(C) 1997 International Press

Adv. Theor. Math. Phys. 1 (1997) 1-52

\title{
Strong Coupling Dynamics of Four-Dimensional $N=1$ Gauge Theories from M-Theory Fivebrane
}

\author{
Kentaro Hori, Hirosi Ooguri, Yaron Oz \\ Department of Physics, University of California at Berkeley \\ 366 Le Conte Hall, Berkeley, CA 94720-7300, U.S.A. \\ and \\ Theoretical Physics Group, Mail Stop 50A-5101 \\ Ernest Orlando Lawrence Berkeley National Laboratory \\ Berkeley, CA 94720, U.S.A.
}

\begin{abstract}
It has been known that the fivebrane of type IIA theory can be used to give an exact low energy description of $N=2$ supersymmetric gauge theories in four dimensions. We follow the recent M-theory description by Witten and show that it can be used to study theories with $N=1$ supersymmetry. The $N=2$ supersymmetry can be broken to $N=1$ by turning on a mass for the adjoint chiral superfield in the $N=2$ vector multiplet. We construct the configuration of the fivebrane for both finite and infinite values of the adjoint mass. The fivebrane describes strong coupling dynamics of $N=1$ theory with $S U\left(N_{c}\right)$ gauge group and $N_{f}$ quarks. For $N_{c}>N_{f}$, we show how the brane configuration encodes the information of the Affleck-Dine-Seiberg superpotential. For $N_{c} \leq N_{f}$, we study the deformation space of the brane configuration and compare it with the moduli space of the $N=1$ theory. We find agreement with field theory results, including the quantum deformation of the moduli space at $N_{c}=N_{f}$. We also prove the type II $s$-rule in M-theory and find new non-renormalization theorems for $N=1$ superpotentials.
\end{abstract}




\section{Introduction}

In the past few years, we have learnt much about non-perturbative dynamics of supersymmetric gauge theories and string theories. In particular, the $\mathrm{D}$ (irichlet)-brane [1] has provided an arena to exchange results of gauge theories and string theories and to advance our knowledge of both. This approach is very profitable since the gauge coupling constant and the string coupling constant are in general different. Therefore perturbative results in one theory can be translated into non-perturbative statements in the other theory. For example, if we compactify the type II string on a singular CalabiYau three-fold and turn off the gravity, we obtain an $N=2$ gauge theory in four dimensions. In this case, the coupling constant of the gauge theory is some geometric modulus of the three-fold, totally independent of the string coupling constant [2-4]. Thus strong coupling dynamics of the gauge theory can be translated into facts about the geometry of the three-fold.

One can also obtain gauge theories in four dimensions by considering webs of NS 5-branes and D4-branes in a flat space in the type IIA string theory ${ }^{1}$. A typical configuration consists of two parallel NS 5-branes and several D4-branes suspended between them. If world-volumes of the 5- and 4-branes share four flat dimensions, we obtain $N=2$ gauge theory in four dimensions with $S U\left(N_{c}\right)$ gauge group, where $N_{c}$ is the number of D4-branes. We can add $N_{f}$ pairs of quark chiral multiplets in the fundamental representation of $S U\left(N_{c}\right)$ by attaching $N_{f}$ semi-infinite D4-branes to one of the NS 5-branes.

In this construction, the distances between the $N_{c}$ D4-branes suspended between the NS 5-branes correspond to the vacuum expectation values (vevs) of the adjoint scalar field in the vector multiplet and therefore parametrize the Coulomb branch of the model. If we turn on a mass of this adjoint field, the $N=2$ supersymmetry is broken to $N=1$. This corresponds to changing the relative orientation of the two NS 5-branes while leaving their common four dimensions intact [7]. If the NS 5-branes are not parallel, the position of the D4-branes is fixed in order to minimize their world-volume. Thus the Coulomb branch is lifted. In the limit when the relative angle of the two NS 5 -branes becomes $\pi / 2$, the adjoint mass becomes infinite and we obtain the $N=1$ gauge theory with $S U\left(N_{c}\right)$ gauge group with $N_{f}$ quarks. This is the configuration studied in $[8,9]$.

Recently Witten showed that one can give an exact low energy description of the $N=2$ theory by reinterpreting the brane configuration from the point of view of M-theory [10]. It is known that both D4-branes and

\footnotetext{
${ }^{1}$ This is the T-dual of the configuration first introduced in [5] to study aspects of $N=4$ gauge theories in three dimensions. Configurations of intersecting branes have also been used in order to count the microscopic degrees of freedom of black holes with various amounts of supersymmetry [6].
} 
NS 5-branes of the type IIA theory come from the fivebranes of M-theory, wrapped or unwrapped respectively on the eleventh dimensional circle with radius $R$. Thus the web of the D4-branes and the NS 5-branes in the above may be considered as an $R \rightarrow 0$ limit of a smooth configuration of a single fivebrane in M-theory. The $N=2$ supersymmetry in four dimensions requires that the world-volume of the fivebrane is $\mathbb{R}^{1,3} \times \Sigma$ and that $\Sigma$ is holomorphically embedded in the $\mathbb{R}^{3} \times S^{1}$ part of the eleven dimensions [11] 2. Witten has shown that, by imposing appropriate boundary conditions, the configuration of $\Sigma$ is uniquely determined as

$$
t^{2}-C_{N_{c}}\left(v, u_{k}\right) t+\Lambda_{N=2}^{2 N_{c}-N_{f}} \prod_{i=1}^{N_{f}}\left(v+m_{i}\right)=0,
$$

where $(v, t)$ are holomorphic coordinates on $\mathbb{R}^{3} \times S^{1}, C_{N_{c}}\left(v, u_{k}\right)$ is a polynomial in $v$ of degree $N_{c}$ with coefficients which depend on the moduli $u_{k}$, and $m_{i}\left(i=1, \ldots, N_{f}\right)$ are the masses of the quarks.

In the M-theory description, the coupling constant of the type IIA string is given by $g_{s}^{2} \sim\left(R / l_{11}\right)^{3}$ where $l_{11}$ is the eleven-dimensional Planck scale, while the four-dimensional gauge coupling constant scales as $g_{\text {gauge }}^{2} \sim$ $R / L_{\text {brane }}$, where $L_{\text {brane }}$ is a characteristic size of the brane such as the distance between the NS 5-branes in the type IIA picture. Therefore we can take the limit, $R, L_{\text {brane }} \gg l_{11} \rightarrow 0$, while keeping the gauge coupling constant finite. Since the eleven-dimensional supergravity gives the low energy description of M-theory, in this scaling limit the configuration (1.1) should capture the strong coupling dynamics of the four-dimensional gauge theory. In fact, it turns out that $\Sigma$ given by (1.1) is the same as the Riemann surface that appears in the exact solution to the $N=2$ gauge theory [14-18].

Here we would like to make some historical remarks. It has been pointed out earlier in [4] that the Seiberg-Witten curve is geometrically realized as a configuration of the 5-brane. They considered the NS 5-brane in the type IIA string theory rather than the fivebrane of M-theory. However, at least in the case where there is $N=2$ supersymmetry in four dimensions, these two are essentially the same object.

The purpose of this paper is to study the $N=1$ gauge theory, which is obtained by turning on the adjoint mass in the $N=2$ theory, using the fivebrane. We identify the fivebrane configuration for non-zero value of the adjoint mass. As we mentioned before, the adjoint mass lifts the Coulomb branch of the $N=2$ theory except at the roots of the Higgs branches. Correspondingly we find that in order to rotate the fivebrane we have to tune its moduli $u_{k}$ to completely degenerate the curve $\Sigma$ and make it birationally

\footnotetext{
${ }^{2}$ Four-dimensional abelian theory obtained from a fivebrane on $\mathbb{R}^{1,3} \times \Sigma$ was studied in [12]. A related observation was also made in [13].
} 
equivalent to $\mathbb{C P}^{1}$. We then show that there is a unique way to rotate the fivebrane and determine how it is embedded in the eleven dimensions.

In the $N=1$ theory, a superpotential is non-perturbatively generated for $N_{c}>N_{f}$ [19] and there are no supersymmetric vacua. If we add quark masses and/or a perturbation quartic in the quark chiral superfields that corresponds to the adjoint mass in the $N=2$ theory, the theory has vacua characterized by the vevs of $\tilde{Q}_{i}^{a} Q_{a}^{j}$, where $Q_{a}^{i}, \tilde{Q}_{i}^{a}$ are the squark fields $\left(i=1, \ldots, N_{f}, a=1, \ldots, N_{c}\right)$. In the type IIA picture, these vevs are related to locations of the semi-infinite D4-brane. We compute locations of the corresponding branches of the M-theory fivebrane and show that they are in complete agreement with the values of $\tilde{Q}_{i}^{a} Q_{a}^{j}$ obtained by the field theory method, as functions of the quark and the adjoint masses.

When $N_{c} \leq N_{f}$, there is an additional moduli space associated with the configuration of the fivebrane that corresponds to a complete Higgsing of the gauge group. We study the structure of the moduli space and compare it with that for the $N=1$ theory. We also use the fivebrane to obtain nonrenormalization theorems that have not been proven via the standard field theory method.

This paper is organized as follows:

Section 2 is devoted to field theory analysis. We study the moduli space of vacua of the $N=1$ theory which is obtained from the $N=2$ theory by adding a mass term to the adjoint chiral multiplet. In particular we calculate the minima of the $N=1$ superpotential which, for $N_{c}>N_{f}$, consists of the Affleck-Dine-Seiberg superpotential, a quartic perturbation in the quark chiral superfields and a quark mass term. We prove a non-renormalization theorem of such a superpotential and show that its minima are precisely as expected if we start with the $N=2$ theory and add a mass to the adjoint field. We perform similar analysis when $N_{f}=N_{c}$ and when $N_{f} \geq N_{c}+1$, where in the latter case we make use of the magnetic dual theory.

In section 3, we develop techniques to study the moduli space of vacua using the fivebrane of M-theory. In particular we show in detail how the Higgs branches of the $N=2$ theory are described in this language.

In section 4, we show how to rotate the fivebrane to break the $N=2$ supersymmetry to $N=1$ and present the resulting configuration explicitly for non-zero value of the adjoint mass. We find that the configuration encodes the strong coupling dynamics of the $N=1$ theory. Specifically, we read vevs of the mesons parametrizing the Higgs branches from the brane configuration and show that they are in complete agreement with the field theory results of section 2 .

In section 5 , we take the limit where the mass of the adjoint is infinite. In the case of $N_{c} \leq N_{f}$, we study the deformation space of the brane configuration and compare it with the moduli space of vacua of the $N=1$ theory. 
Again we find a complete agreement with the field theory results.

In Section 6 we further study the moduli space of vacua of the $N=1$ theory. We find how the baryons are realized in the M-theory fivebrane and find again a complete agreement with the field theory results of section 2 . In this section we also prove the $s$-rule of [5].

In section 7 we comment on the Kähler potential in the M-theory framework.

We note that the method of intersecting branes in IIA and IIB string theories and in some cases their M-theory description have been applied to the study of supersymmetric field theories in various dimensions in [22-36]

\section{$2 \quad$ Field Theory Analysis}

In this section we analyse in the field theory framework the moduli space of vacua obtained by breaking $N=2$ to $N=1$ by adding a mass term to the adjoint chiral multiplet.

\section{1 $N=2$ Moduli Space of Vacua}

We consider $N=2$ supersymmetric gauge theory with $S U\left(N_{c}\right)$ gauge group and $N_{f}$ quark hypermultiplets in the fundamental representation. In terms of $N=1$ superfields the vector multiplet consists of a field strength chiral multiplet $W_{\alpha}$ and a scalar chiral multiplet $\Phi$, both in the adjoint representation of the gauge group. A quark hypermultiplet consists of a chiral multiplet $Q$ in the $N_{c}$ and $\tilde{Q}$ in the $\bar{N}_{c}$ representation of the gauge group. The $N=2$ superpotential takes the form

$$
W=\sqrt{2} \tilde{Q}_{i}^{a} \Phi_{a}^{b} Q_{b}^{i}+\sqrt{2} m_{j}^{i} \tilde{Q}_{i}^{a} Q_{a}^{j},
$$

where $a, b=1, \ldots, N_{c} ; i, j=1, \ldots, N_{f}$ and the quark mass matrix $m=$ $\operatorname{diag}\left[m_{1}, \ldots, m_{N_{f}}\right]$.

The $R$-symmetry group is $S U(2)_{R} \times U(1)_{R}$. The bosons in the vector multiplet are singlets under $S U(2)_{R}$ while the fermions in the vector multiplet form a doublet. The fermions in the hypermultiplet are singlets under $S U(2)_{R}$ while the scalars in the hypermultiplet form a doublet. The theory is asymptotically free for $N_{f}<2 N_{c}$. The instanton factor is proportional to $\Lambda^{2 N_{c}-N_{f}}$ where $\Lambda$ is the dynamically generated scale. The $U(1)_{R}$ symmetry is anomalous and is broken to $\mathbb{Z}_{2 N_{c}-N_{f}}$.

The moduli space of vacua includes the Coulomb and Higgs branches. The Coulomb branch is $N_{c}-1$ complex dimensional and is parametrized by the gauge invariant order parameters

$$
u_{k}=\left\langle\operatorname{Tr}\left(\phi^{k}\right)\right\rangle, \quad k=2, \ldots, N_{c}
$$


where $\phi$ is the scalar field in the vector multiplet. Generically along the Coulomb branch the gauge group is broken to $U(1)^{N_{c}-1}$. The Coulomb branch structure is corrected by one loop effects and by instantons. The quantum Coulomb branch parametrizes a family of genus $N_{c}-1$ hyperelliptic curves whose period matrix $\tau_{i j}$ is the low energy gauge coupling [14-18].

Two types of Higgs branches are distinguished [38]: The baryonic branch and the non- baryonic branches. There is a single baryonic branch for $N_{f} \geq N_{c}$, where generically the gauge group is completely broken. Its complex dimension is $2 N_{f} N_{c}-2\left(N_{c}^{2}-1\right)$. The non-baryonic branches are classified by an integer $r$ such that $1 \leq r \leq \min \left\{\left[N_{f} / 2\right], N_{c}-2\right\}$. The $r$-th non-baryonic branch has complex dimension $2 r\left(N_{f}-r\right)$. The baryonic branch emanates from a point in the Coulomb branch while the non-baryonic branches emanate from submanifolds in the Coulomb branch (of dimension $N_{c}-r-1$ for the $r$-th non-baryonic branch) and constitute mixed branches. The Higgs branches are determined classically; however, where they intersect with each other and with the Coulomb branch is modified quantum mechanically.

\subsection{Breaking $N=2$ to $N=1$}

The $N=2$ supersymmetry is broken to $N=1$ by turning on a bare mass $\mu$ for the adjoint chiral multiplet $\Phi$

$$
W=\sqrt{2} \operatorname{Tr}(\widetilde{Q} \Phi Q)+\mu \operatorname{Tr}\left(\Phi^{2}\right) .
$$

When the mass for the adjoint chiral multiplet is small we can still use the low energy description of [14], and it turns out that the structure of the moduli space of vacua is modified as follows. Most of the Coulomb branch is lifted except for a discrete set of points. $2 N_{c}-N_{f}$ points related to each other by the action of $\mathbb{Z}_{2 N_{c}-N_{f}}$ and which correspond to to points in the moduli space of vacua where all the $\alpha$ cycles of the hyper-elliptic curve vanish remain. The root of the baryonic branch as well as the baryonic branch itself remain. The non-baryonic branches remain but instead of being mixed branches they emanate from points. More precisely, the $r$-th non-baryonic branch that emanated from a submanifold of dimension $N_{c}-r-1$ in the Coulomb branch is now emanating from $2 N_{c}-N_{f}$ points related to each other by $\mathbb{Z}_{2 N_{c}-N_{f}}$ with the exception of the $r=N_{f} / 2$ case $\left(N_{f}\right.$ even) where the $\mathbb{Z}_{2}$ subgroup is unbroken and the $\mathbb{Z}_{2 N_{c}-N_{f}}$ orbit consists of $N_{c}-N_{f} / 2$ points.

When the mass $\mu$ for the adjoint chiral multiplet is increased beyond $\Lambda_{N=2}$, the renormalization group flow below the scale $\mu$ is the same as in $N=1$ Supersymmetric QCD (SQCD) with the dynamical scale $\Lambda_{N=1}$ given by

$$
\Lambda_{N=1}^{3 N_{c}-N_{f}}=\mu^{N_{c}} \Lambda_{N=2}^{2 N_{c}-N_{f}} .
$$


If $\mu$ is much larger than $\Lambda_{N=1}$ but finite, we can first integrate out the heavy field $\Phi$ in (2.3), obtaining a superpotential which is quartic in the quark chiral superfields and is proportional to $1 / \mu$ :

$$
\Delta W=\frac{1}{2 \mu}\left(\operatorname{Tr}\left(M^{2}\right)-\frac{1}{N_{c}}(\operatorname{Tr} M)^{2}\right),
$$

where $M=\widetilde{Q} Q$. In other words, we may consider the system below the energy scale $\mu$ as the $N=1 \mathrm{SQCD}$ with the tree level superpotential $\Delta W$ and the dynamical scale $\Lambda_{N=1}$ given by (2.4). As we send the mass $\mu$ to infinity keeping $\Lambda_{N=1}$ finite, the potential $\Delta W$ disappears and the system becomes equivalent to $N=1 \mathrm{SQCD}$, whose low energy properties depend on the number of flavors $N_{f}$. The structure of the moduli space of vacua should match for finite values of $\mu$ with the one that we get by starting with $N=2$ and adding mass for the adjoint chiral multiplet. This will be checked in the following.

Pure Yang-Mills Theory $N_{f}=0$

For $N=1 S U\left(N_{c}\right)$ Yang-Mills theory, there are $N_{c}$ massive vacua where the discrete $\mathbb{Z}_{2 N_{c}} R$-symmetry is spontaneously broken to $\mathbb{Z}_{2}$, as the computation of the Witten index [37] shows. They correspond to $N_{c}$ curves in the $N=2$ theory with all the $\alpha$ cycles vanishing. These curves are related by the action of the discrete $\mathbb{Z}_{2 N_{c}} R$-symmetry group, which is consistent with the structure of the $N=1$ vacua.

$0<N_{f}<N_{c}$

In $N=1 \mathrm{SQCD}$ with the the number of flavors in this region, a superpotential is dynamically generated [19]: for $N_{f}=N_{c}-1$ it is due to the effect of instantons, and for the other cases it is due to a strong gauge dynamics. It takes the form

$$
W_{\mathrm{ADS}}=\left(N_{c}-N_{f}\right)\left(\frac{\Lambda_{N=1}^{3 N_{c}-N_{f}}}{\operatorname{det} M}\right)^{1 /\left(N_{c}-N_{f}\right)},
$$

and thus there is no supersymmetric vacuum.

For large but finite $\mu$, at the scale far below $\mu$ but much larger than $\Lambda_{N=1}$, the quartic term is very small and can be considered as a perturbation to the ordinary $N=1$ system. Therefore the superpotential (2.6) is generated in this case as well. Thus, we expect that the effective superpotential is just the sum

$$
\begin{aligned}
W_{\text {eff }} & =\left(N_{c}-N_{f}\right)\left(\frac{\Lambda_{N=1}^{3 N_{c}-N_{f}}}{\operatorname{det} M}\right)^{1 /\left(N_{c}-N_{f}\right)}+ \\
& +\frac{1}{2 \mu}\left(\operatorname{Tr}\left(M^{2}\right)-\frac{1}{N_{c}}(\operatorname{Tr} M)^{2}\right) .
\end{aligned}
$$


In fact, this is an exact superpotential which is valid for any non-zero value of $\mu$. This follows from the following holomorphy argument [39]. The superpotential must be an analytic function around the decoupling limit $1 / \mu=0$ and therefore can be expanded with respect to $1 / \mu$ where the first two terms are fixed to be (2.7). Thus, a term that can be generated takes the form

$$
\mu^{-\alpha} M^{\beta} \Lambda_{N=1}^{\left(3 N_{c}-N_{f}\right) \gamma},
$$

where $\alpha$ is a non-negative integer and $M^{\beta}$ is some combination of the meson matrix of order $\beta$ which is invariant under the flavor group $S U\left(N_{f}\right)$. We require $\gamma \geq 0$ for the existence of the weak coupling limit $\Lambda_{N=1} \rightarrow 0$. We recall that $\mu, M$, and $\Lambda^{3 N_{c}-N_{f}}$ carry the following $U(1)_{R} \times U(1)_{A}$ charges where $U(1)_{R}$ is the anomaly free combination of the $U(1) R$-symmetry group, while $U(1)_{A}$ is the axial flavor symmetry which is anomalously broken.

$$
\begin{array}{cccc} 
& \Lambda_{N=1}^{3 N_{c}-N_{f}} & M & \mu \\
U(1)_{R} & 0 & 2 \frac{N_{f}-N_{c}}{N_{f}} & 2 \frac{N_{f}-2 N_{c}}{N_{f}} \\
U(1)_{A} & 2 N_{f} & 2 & 4
\end{array} .
$$

The charges of $\mu$ are determined so that the perturbation term $\int \mathrm{d}^{2} \theta \Delta W$ is invariant, and the $U(1)_{A}$ charge of the instanton factor $\Lambda_{N=1}^{3 N_{c}-N_{f}}$ reflects the axial anomaly. The perturbation term by (2.8) must be invariant under $U(1)_{R} \times U(1)_{A}$ with this assignment of the charges, and this requires $N_{f}(-\alpha+\beta-1)=N_{c}(-2 \alpha+\beta)$ and $-2 \alpha+\beta=-N_{f} \gamma$, and thus in particular

$$
1-\alpha=\left(N_{c}-N_{f}\right) \gamma
$$

Since we are considering the case $N_{f}<N_{c}$, this together with $\gamma \geq 0$ requires $\alpha=0, \gamma=1 /\left(N_{c}-N_{f}\right)$ or $\alpha=1, \gamma=0$. The former corresponds to the Affleck-Dine-Seiberg potential (2.6), and the latter corresponds to the tree level term $\Delta W$. In this way, we have seen that the superpotential (2.7) is exact.

The moduli space of vacua is the variety of extrema of this superpotential. We now determine this. Extremizing (2.7) we have

$$
M^{2}-\frac{1}{N_{c}}(\operatorname{Tr} M) M=\mu\left(\frac{\Lambda_{N=1}^{3 N_{c}-N_{f}}}{\operatorname{det} M}\right)^{1 /\left(N_{c}-N_{f}\right)} .
$$

Let us perform a similarity transformation $M \rightarrow M^{\prime}=g M g^{-1}$ in (2.11) such that $M_{i+j, i}^{\prime}=0, j>0$ and define $m_{i}:=M_{i i}$. It is not possible for more than two of the elements $m_{i}$ to have different values. In order to see this 
suppose that there are three different values $m_{i}, m_{j}, m_{k}$. Equation (2.11) for the diagonal elements

$$
m_{i}^{2}-\frac{1}{N_{c}}\left(\sum_{l=1}^{N_{f}} m_{l}\right) m_{i}=\mu\left(\frac{\Lambda_{N=1}^{3 N_{c}-N_{f}}}{\prod_{l} m_{i}}\right)^{1 /\left(N_{c}-N_{f}\right)}
$$

implies upon subtracting the equations for any two of $m_{i}, m_{j}, m_{k}$, that

$$
m_{i}+m_{j}=m_{j}+m_{k}=m_{i}+m_{k},
$$

in contradiction with the assumption that they all have different values.

In fact $M$ can be diagonalized. In order to see that assume that this is not the case. Then $M$ can be brought to the Jordan form. In this case we will get from off diagonal entries in (2.11)

$$
2 m_{1}-\frac{1}{N_{c}} \operatorname{Tr} M=0 .
$$

If we assume that there exists another diagonal entry value $m_{2} \neq m_{1}$ we will also get an equation of the form

$$
m_{1}+m_{2}-\frac{1}{N_{c}} \operatorname{Tr} M=0,
$$

in contradiction with (2.14). The Jordan form implies that all the diagonal entries must be the same and therefore (2.14) yields $2 m_{1}=m_{1} N_{f} / N_{c}$, which cannot be satisfied since $N_{f}<N_{c}$. We are led to a contradiction and thus $M$ can be diagonalized.

Since there are no more than two possible values for the diagonal entries we find two types of solutions to (2.12). In the first type all the diagonal entries are equal, $m_{1}=\ldots=m_{N_{f}}=m$, where

$$
m=\left(\frac{N_{c}}{N_{c}-N_{f}}\right)^{\frac{N_{c}-N_{f}}{2 N_{c}-N_{f}}} \mu \Lambda_{N=2},
$$

and we used the RG matching relation (2.4). This solution corresponds in the $N=2$ picture to the case when all the $\alpha$ cycles degenerate, and will be denoted as the $r=0$ case. In the second type there are two different entries on the diagonal. They can be made to take the form $m_{1}=\ldots=m_{r}=$ $m_{(1)} \neq m_{r+1}=\ldots=m_{N_{f}}=m_{(2)}$. The equations determining $m_{(1)}$ and $m_{(2)}$ are

$$
\begin{aligned}
& \left(1-\frac{r}{N_{c}}\right) m_{(1)}+\left(1-\frac{N_{f}-r}{N_{c}}\right) m_{(2)}=0 \\
& m_{(1)}^{N_{c}-N_{f}+r} m_{(2)}^{N_{c}-r}=(-1)^{N_{c}-N_{f}}\left(\mu \Lambda_{N=2}\right)^{2 N_{c}-N_{f}}
\end{aligned}
$$


and the solution is

$$
m_{(2)}=\left((-1)^{r}\left(\frac{N_{c}-r}{N_{c}-N_{f}+r}\right)^{N_{c}-N_{f}+r}\right)^{\frac{1}{2 N_{c}-N_{f}}} \mu \Lambda_{N=2} .
$$

where $m_{(1)}$ is given by $(2.17)$.

To summarize: The solutions (2.19) are classified by an integer $r$ that takes values in the range $0 \leq r \leq\left[N_{f} / 2\right]$. The moduli space consists of the orbits of the complexified flavor group $G L\left(N_{f}, \mathbb{C}\right)$ through such diagonal solutions. Since the diagonal solution for $r$ is invariant under the subgroup $G L(r, \mathbb{C}) \times G L\left(N_{f}-r, \mathbb{C}\right)$, the moduli space is the homogeneous space $G L\left(N_{f}, \mathbb{C}\right) /\left(G L(r, \mathbb{C}) \times G L\left(N_{f}-r, \mathbb{C}\right)\right)$, which has complex dimension $2 r\left(N_{f}-r\right)$. This dimension agrees with what we expect from the $N=2$ discussion where $r$ is the parameter that characterizes the non-baryonic branches. As we see from the solution (2.19), for each $r<N_{f} / 2$ there are $2 N_{c}-N_{f}$ solutions related by the action of $\mathbb{Z}_{2 N_{c}-N_{f}}$, as expected. For $r=N_{f} / 2\left(N_{f}\right.$ even), however, since $m_{(1)}=-m_{(2)}$, the two solutions related by the $\mathbb{Z}_{2}$ subgroup (sign change) are related by conjugation by an element of $G L\left(N_{f}, \mathbb{C}\right)$, and thus are in the same orbit. So there are only $N_{c}-N_{f} / 2$ families, which is also expected.

In the limit $\mu \rightarrow \infty$ keeping $\Lambda_{N=1}$ finite, all these solutions diverge since $\left(\mu \Lambda_{N=2}\right)^{2 N_{c}-N_{f}}=\mu^{N_{c}-N_{f}} \Lambda_{N=1}^{3 N_{c}-N_{f}}$. This is consistent with the fact that there is no supersymmetric vacuum for the $N=1$ SQCD in this region of the flavor.

\section{Inclusion of Bare Mass}

Let us consider the case where the quark mass term $\sum\left(m_{f}\right)_{i, j} \widetilde{Q}^{i} Q_{j}$ is turned on. The effective superpotential is given by

$$
W_{\text {eff }}=W_{\mathrm{ADS}}+\Delta W+\operatorname{Tr}\left(m_{f} M\right),
$$

where $W_{\mathrm{ADS}}$ and $\Delta W$ are given by (2.6) and (2.5). Again, this is an exact superpotential as can be seen from the analyticity at $m_{f}=1 / \mu=0$ and charge conservation, where $m_{f}$ carries $U(1)_{R} \times U(1)_{A}$ charge $\left(2 N_{c} / N_{f},-2\right)$.

Extremizing $W_{\text {eff }}$, we obtain the moduli space of vacua. Here we present the result in the case where the mass matrix is proportional to the identity matrix, $\left(m_{f}\right)_{i, j}=m_{f} \delta_{i, j}$. In this case, as in the previous discussion, $M$ is diagonalizable ${ }^{3}$, and there are at most two kinds of eigenvalues. Thus, it is again classified by $r=0,1, \ldots,\left[N_{f} / 2\right]$. The equations determining the two (or one in the case $r=0$ ) eigenvalues $m_{1}=\cdots=m_{r}=m_{(1)}$ and

\footnotetext{
${ }^{3}$ For some special values of $m_{f}$ a Jordan block of size two is allowed.
} 
$m_{r+1}=\cdots=m_{N_{f}}=m_{(2)}$ are in this case

$$
\begin{aligned}
& \left(1-\frac{r}{N_{c}}\right) m_{(1)}+\left(1-\frac{N_{f}-r}{N_{c}}\right) m_{(2)}+\mu m_{f}=0, \\
& m_{(1)}^{N_{c}-N_{f}+r} m_{(2)}^{N_{c}-r}=(-1)^{N_{c}-N_{f}}\left(\mu \Lambda_{N=2}\right)^{2 N_{c}-N_{f}} .
\end{aligned}
$$

Note that there are $2 N_{c}-N_{f}$ solutions for each $r$, but they are not related any longer by the discrete $R$-symmetry group $\mathbb{Z}_{2 N_{c}-N_{f}}$ which is explicitly broken by the quark mass term.

Lets us consider the limit $\mu \rightarrow \infty$ keeping $\Lambda_{N=1}$ finite where the system becomes the $N=1 \mathrm{SQCD}$ with massive quarks. Since $\left(\mu \Lambda_{N=2}\right)^{2 N_{c}-N_{f}}=$ $\mu^{N_{c}-N_{f}} \Lambda_{N=1}^{3 N_{c}-N_{f}}, m_{(1)}$ or $m_{(2)}$ must diverge in the limit. Because a vacuum must have a finite vev for $M=\widetilde{Q} Q$, only the solutions with $r=0$ and $m_{(2)}$ finite remain as supersymmetric vacua. In this case, $m_{(1)}$ diverges as $m_{(1)} \sim-\mu m_{f}$ as follows from the first equation (2.21). Then, inserting this to the second equation, we see that $M_{j}^{i}=m_{(2)} \delta_{j}^{i}$ where

$$
m_{(2)}^{N_{c}}=\Lambda_{N=1}^{3 N_{c}-N_{f}} / m_{f}^{N_{c}-N_{f}}
$$

in the limit $\mu \rightarrow \infty$. Thus, we have $N_{c}$ vacua. This is consistent with the interpretation of the low energy physics as the pure $N=1$ Yang-Mills theory.

$\underline{N_{f}=N_{c}}$

In $N=1 \mathrm{SQCD}$ with this number of flavors, the classical moduli space of vacua is modified quantum mechanically. It is parametrized by the meson $M=\tilde{Q} Q$ and the baryons $B=Q^{N_{c}}, \tilde{B}=\tilde{Q}^{N_{c}}$ satisfying the constraint [20]

$$
\operatorname{det} M-\tilde{B} B=\Lambda_{N=1}^{2 N_{c}} .
$$

As in the case $N_{f}<N_{c}$, for large finite $\mu$, at the scale far below $\mu$ and much larger than $\Lambda_{N=1}$, the quartic term (2.5) is very small and can be considered as a perturbation to the ordinary $N=1$ system. We expect that the effective superpotential is

$$
W_{e f f}=X\left(\operatorname{det} M-\tilde{B} B-\Lambda_{N=1}^{2 N_{c}}\right)+\frac{1}{2 \mu}\left(\operatorname{Tr}\left(M^{2}\right)-\frac{1}{N_{c}}(\operatorname{Tr} M)^{2}\right),
$$

where we introduced a Lagrange multiplier $X$ to impose the constraint (2.24). In this case the holomorphy and global symmetries are not powerful enough to ensure that (2.25) is the exact superpotential. In section 4, we will see by brane analysis that this is indeed the case. 

to $X$,

Extremizing $W_{\text {eff }}$ we get equation (2.24) from the derivative with respect

$$
X B=0, \quad X \tilde{B}=0,
$$

from the derivative with respect to $\tilde{B}, B$ and

$$
M^{2}-\frac{1}{N_{c}}(\operatorname{Tr} M) M=-\mu X \operatorname{det} M,
$$

from the derivative with respect to $M$.

Consider the last equation. Similar analysis as the one done in the $N_{f}<$ $N_{c}$ case shows that $M$ can be diagonalized and can have at most two different eigenvalues, which as before we denote by $m_{1}=\ldots=m_{r}=m_{(1)} \neq m_{r+1}=$ $\ldots=m_{N_{f}}=m_{(2)}$. There are two cases to consider, $X=0$ and $X \neq 0$. When $X=0$ then for $\tilde{B} B=0$ we get using (2.24) that the solution to (2.27) is given by

$$
m_{(1)}=m_{(2)}=\Lambda_{N=1}^{2},
$$

while for $\tilde{B} B \neq 0$ we have $m_{(1)}=m_{(2)}=m$ related to $\tilde{B} B$ by

$$
m^{N_{c}}-\tilde{B} B=\Lambda_{N=1}^{2 N_{c}} .
$$

When $X \neq 0$ the equations determining $m_{(1)}$ and $m_{(2)}$ are

$$
\begin{aligned}
& \left(1-\frac{r}{N_{c}}\right) m_{(1)}+\left(1-\frac{N_{c}-r}{N_{c}}\right) m_{(2)}=0, \\
& m_{(1)}^{r} m_{(2)}^{N_{c}-r}=\left(\mu \Lambda_{N=2}\right)^{N_{c}}
\end{aligned}
$$

and the solution to (2.31) is given by

$$
\begin{aligned}
& m_{(1)}=\left((-1)^{N_{c}-r}\left(\frac{r}{N_{c}-r}\right)^{N_{c}-r}\right)^{\frac{1}{N_{c}}} \mu \Lambda_{N=2} \\
& m_{(2)}=\left((-1)^{r}\left(\frac{N_{c}-r}{r}\right)^{r}\right)^{\frac{1}{N_{c}}} \mu \Lambda_{N=2}
\end{aligned}
$$

and $B=\tilde{B}=0$ by $(2.26)$.

As in the $N_{f}<N_{c}$ case, the solutions (2.33) correspond to the $r$-th nonbaryonic branch of complex dimension $r$. As we see from the solution (2.33), for each $r$ there are $N_{c}$ solutions related by the action of $\mathbb{Z}_{N_{c}}$. The solution (2.29) corresponds to a new branch which did not exist in the $N_{f}<N_{c}$ region. This is a complex dimension one submanifold of the baryonic branch whose complex dimension is two. This branch corresponds to a complete Higgsing of the gauge group. 
In the limit $\mu \rightarrow \infty$ keeping $\Lambda_{N=1}^{2}$ finite, all these solutions remain with $\mu \Lambda_{N=2}=\Lambda_{N=1}^{2}$ and they define submanifolds of the $N_{c}^{2}+1$ complex dimensional Higgs branch of $N=1$ SQCD.

$\underline{N_{c}+1 \leq N_{f}}$

In this case the classical space of vacua of $N=1 \mathrm{SQCD}$ is not modified quantum mechanically. It is parametrized by the mesons and baryons which are related by a classical constraint. However, it is useful in this case to use the dual magnetic description based on the gauge group $S U\left(N_{f}-N_{c}\right)$ with $N_{f}$ flavors of quarks $q, \tilde{q}$ and gauge invariant fields $M$ with the superpotential [21]

$$
W_{m a g}=\frac{1}{\lambda} \tilde{q} M q+\left(N_{c}-N_{f}\right)\left(\frac{\Lambda_{N=1}^{3 N_{c}-N_{f}}}{\operatorname{det} M}\right)^{1 /\left(N_{c}-N_{f}\right)} .
$$

The scale $\lambda$ relates the scale $\Lambda_{N=1}$ of the electric theory and the scale $\tilde{\Lambda}_{N=1}$ of the magnetic theory by

$$
\Lambda_{N=1}^{3 N_{c}-N_{f}} \tilde{\Lambda}_{N=1}^{3\left(N_{f}-N_{c}\right)-N_{f}}=(-1)^{N_{f}-N_{c}} \lambda^{N_{f}},
$$

and is used in order to relate the electric and magnetic gauge invariant operators. The dimension two (at the UV fixed point) meson $M$ in the electric description is related to the dimension one singlets $M_{m a g}$ in the electric description by $M=\lambda M_{m a g}$. Similarly, the baryons of the electric theory $B, \tilde{B}$ are related to the baryons of the magnetic theory $B_{m a g}, \tilde{B}_{m a g}$ constructed from the dual quarks by $B=C B_{m a g}, \tilde{B}=C \tilde{B}_{m a g}$ with $C=$ $\left(-(-\lambda)^{N_{c}-N_{f}} \Lambda_{N=1}^{3 N_{c}-N_{f}}\right)^{1 / 2}$. Equation (2.33) is written in terms of the electric meson and the scale of the electric theory.

Consider the effective superpotential

$$
W_{\text {eff }}=W_{m a g}+\frac{1}{2 \mu}\left(\operatorname{Tr}\left(M^{2}\right)-\frac{1}{N_{c}}(\operatorname{Tr} M)^{2}\right) .
$$

As in the case $N_{f}=N_{c}$ we do not have a field theory proof that the superpotential (2.35) is exact. The brane picture in section 6 suggests that it is. Let us set the vev of the magnetic quarks to zero. This corresponds to setting the vev of the baryon operator $B, \tilde{B}$ to zero and studying the non-baryonic branches. The superpotential that we get in this case is identical to the one analysed in the region $N_{f}<N_{c}$. Therefore, its extrema are precisely those given by equations (2.16), (2.17), (2.18) and (2.19).

Consider now the case where the vev of the magnetic quarks in (2.35) is different from zero. We will not study the complete moduli space of vacua obtained as the extrema of (2.35) and restrict ourselves to those solutions which will be needed in section 6 for comparison with the M-theory fivebrane. 
We start with $N_{f}=N_{c}+1$. In this case $W_{\text {mag }}$ does not contain a fractional power of $\operatorname{det} M$ and it is straightforward to see that

$$
M=\operatorname{diag}[0, m, \ldots, m], \quad B_{m a g}=(b, 0, \ldots, 0), \quad \tilde{B}_{m a g}^{t}=(\tilde{b}, 0, \ldots, 0),
$$

is a solution to the extrema of $(2.35)$ when

$$
\frac{m^{N_{c}}}{\Lambda_{N=1}^{3 N_{c}-N_{f}}}-\frac{\tilde{b} b}{\lambda}=\frac{m}{\mu}
$$

In terms of the gauge invariant operators of the electric theory (2.37) reads

$$
m^{N_{c}}-\tilde{B} B=\frac{m}{\mu} \Lambda_{N=1}^{3 N_{c}-N_{f}} .
$$

Consider next the region $N_{f}>N_{c}+1$, and the following generalization of $(2.36)$

$$
M=\operatorname{diag}[\overbrace{0, . ., 0}^{N_{f}-N_{c}}, \overbrace{m, \ldots, m}^{N_{c}}], \quad q_{i j}=q_{i} \delta_{i j} \quad \tilde{q}_{i j}=\tilde{q}_{i} \delta_{i j},
$$

where we recall that the magnetic quarks $q, \tilde{q}$ are $N_{f} \times\left(N_{f}-N_{c}\right)$ and $\left(N_{f}-N_{c}\right) \times N_{f}$ matrices respectively.

In order to find the extrema of (2.35) we have to specify how to take a limit to approach the region $\operatorname{det} M=0$. In fact, there is an ambiguity in defining the limit. One way to take the limit is to set $M_{k k}=\varepsilon e^{-\frac{2 \pi i k}{N_{f}-N_{c}}}$, $k=1, \ldots, N_{f}-N_{c}$, and take $\varepsilon$ to zero. Extremizing (2.35) we obtain

$$
e^{\frac{2 \pi i k}{N_{f}-N_{c}}}\left(\frac{m^{N_{c}}}{\Lambda_{N=1}^{3 N_{c}-N_{f}}}\right)^{\frac{1}{N_{f}-N_{c}}}-\frac{\tilde{q}_{k} q_{k}}{\lambda}=\frac{m}{\mu}, \quad k=1, \ldots, N_{f}-N_{c} .
$$

Taking the product of equations (2.40) and using the gauge gauge invariant operators of the electric theory, this becomes

$$
m^{N_{c}}-\tilde{B} B=\mu^{N_{c}-N_{f}} m^{N_{f}-N_{c}} \Lambda_{N=1}^{3 N_{c}-N_{f}} .
$$

If we approach $\operatorname{det} M=0$ in a different direction, we obtain a different relation between $m$ and $\tilde{B} B$. As we see later, there is no ambiguity of this type in the fivebrane description. In fact, the fivebrane chooses this particular way to take the limit. We will discuss more on this issue in section 6 .

We close this section with several comments. The baryonic branch which is the branch that includes non zero vev for the baryon operator $B, \tilde{B}$ has complex dimension $2 N_{f} N_{c}-2\left(N_{c}^{2}-1\right)$ for finite $\mu$, and we explored only 
part of it. In the limit $\mu \rightarrow \infty$ keeping $\Lambda_{N=1}$ finite, the solutions (2.16), (2.17) and (2.19) vanish since $\left(\mu \Lambda_{N=2}\right)^{2 N_{c}-N_{f}}=\mu^{N_{c}-N_{f}} \Lambda_{N=1}^{3 N_{c}-N_{f}}$. This is consistent with the fact that on the moduli space of vacua of $N=1$ SQCD with $N_{f} \geq N_{c}+1, \operatorname{det} M=0$ when $\tilde{B} B=0$. The solutions (2.38) and (2.41) in this limit describe part of the moduli space of vacua of $N=1$ SQCD where $\tilde{B} B \neq 0$. In the limit $\mu \rightarrow \infty$ extra $N_{c}^{2}-1$ complex degrees of freedom become massless and the Higgs branch of the theory is $2 N_{c} N_{f}-\left(N_{c}^{2}-1\right)$ complex dimensional with the baryonic and non-baryonic branches being submanifolds of it.

\section{$3 \quad N=2$ Higgs Branch via M-Theory Fivebranes}

In this section we analyse the moduli space of vacua of $N=2$ SQCD by using fivebranes in M-theory. In particular, we study how the Higgs branch of the system is geometrically realized in this picture. This provides the starting point for the studies presented in the following sections.

Let us first review the description of the Higgs branch in the type IIA picture. Consider the brane configuration of [10] that preserves eight supercharges in type IIA string theory on a flat space-time with time $x^{0}$ and space coordinates $x^{1}, \ldots, x^{9}$. The brane configuration depicted in figure 1 consists of two NS 5-branes with worldvolume coordinates $x^{0}, x^{1}, x^{2}, x^{3}, x^{4}, x^{5}, N_{c}$ D4-branes suspended between them with worldvolume coordinates $x^{0}, x^{1}, x^{2}$, $x^{3}, x^{6}$ and $N_{f}$ D6-branes with worldvolume coordinates $x^{0}, x^{1}, x^{2}, x^{3}, x^{7}, x^{8}$, $x^{9}$. Since the D4-brane is finite in the $x^{6}$ direction, the low energy effective theory on the web of branes is the four-dimensional $N=2$ supersymmetric gauge theory on its worldvolume coordinates $x^{0}, x^{1}, x^{2}, x^{3}$. The theory has $S U\left(N_{c}\right)$ gauge group and $N_{f}$ hypermultiplets in the fundamental representation of the gauge group.

Figure 1 depicts the Coulomb branch of the theory. To go to the Higgs branch, we break the D4-branes on the D6-branes and have them suspended between the D6-branes. Motion of the D4-branes along the D6-branes describes the Higgs branch. The location of a D4-brane between two D6-branes is parametrized by two complex parameters, the $x^{7}, x^{8}, x^{9}$ coordinates together with the gauge field component $A_{6}$ in the $x^{6}$ coordinate. Let us count the dimensions of the baryonic and non-baryonic branches in this type IIA picture.

The $r$-th non-baryonic branch, as depicted in figure 2, corresponds to $\left(N_{c}-r\right)$ D4-branes suspended between the two NS 5-branes and $r$ D4-branes broken on the D6-branes.

Since the $s$-rule [5] does not allow more than one D4-brane to be suspended between a NS 5-brane and a D6-brane, $r$ cannot be greater than 

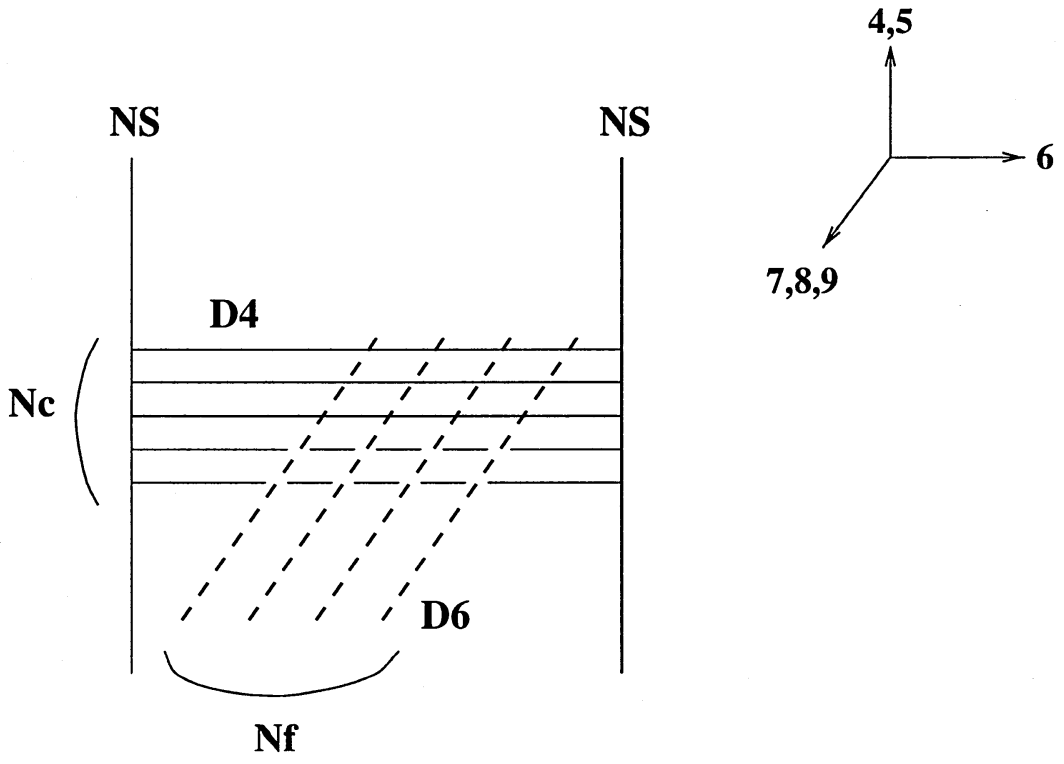

Figure 1: The type IIA picture of $N=2$ supersymmetric $S U\left(N_{c}\right)$ gauge theory with $N_{f}$ flavors (Coulomb branch).

$\left[N_{f} / 2\right]$. Since the Coulomb branch in the brane picture correponds to D4-branes moving along the two NS 5-branes, the $r$-th non-baryonic branch shares $\left(N_{c}-r-1\right)$ complex dimensions with the Coulomb branch, corresponding to gauge group $S U\left(N_{c}-r\right)$. The complex dimension of the non-baryonic branch in the Higgs direction is determined by counting the number of D4branes suspended between the D6-branes. Taking into account the $s$-rule, we obtain the dimension to be

$$
2\left(\left(N_{f}-1\right)+\left(N_{f}-3\right)+\ldots+\left(N_{f}-2 r+1\right)\right)=2 r\left(N_{f}-r\right),
$$

in agreement with the field theory results.

The baryonic branch corresponds to complete Higgsing as in figure 3 . In this case counting the number of D4-brane pieces between two D6-branes yields a complex dimension $2 N_{f} N_{c}-2 N_{c}^{2}$ for the baryonic branch. This is not the correct dimension. Compared to the field theory result, we are missing 2 complex moduli. We will show how the M-theory fivebrane description accounts for these missing moduli.

The brane configuration can be reinterpreted in M-theory as a configuration of a single fivebrane with worldvolume $R^{4} \times \Sigma$ where $\Sigma$ is some genus $\left(N_{c}-1\right)$ curve (Riemann surface). It was shown in [10] that $\Sigma$ is nothing but the Seiberg-Witten curve [14] that determines the structure of the Coulomb branch of the $N=2$ theory. The information on the meromorphic one-form 


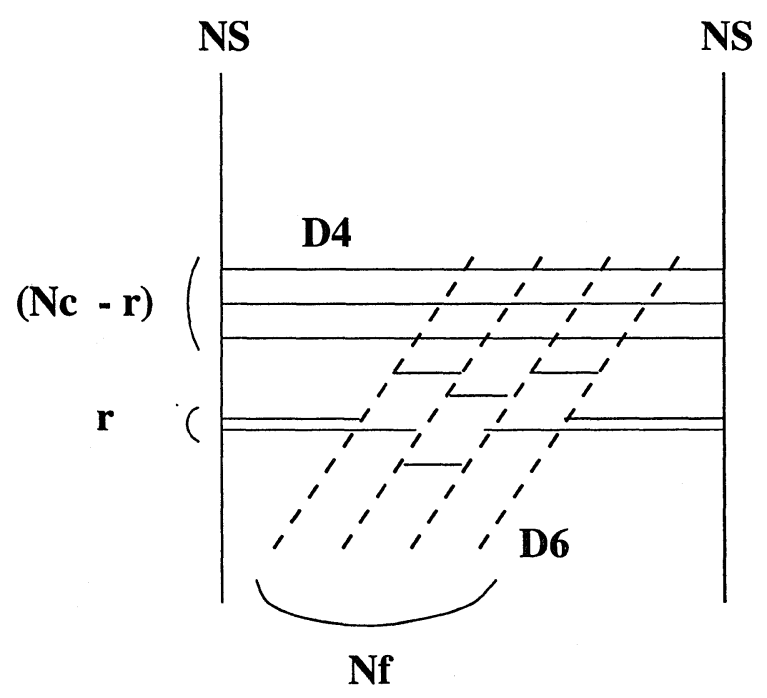

Figure 2: The $r$-th non-baryonic branch in the type IIA picture.

(the Seiberg-Witten form) on $\Sigma$ is carried by the embedding of $\Sigma$ in the space-time.

We follow the notation of [10] and set $v=x^{4}+i x^{5}, s=\left(x^{6}+i x^{10}\right) / R$, $t=\exp (-s)$ where $x^{10}$ is the eleventh coordinate of M-theory which is compactified on a circle of radius $R$. The curve $\Sigma$ is given by an algebraic equation in $(v, t)$ space. Specifically, for $N=2 S U\left(N_{c}\right)$ gauge theory with $N_{f}$ flavors, it is given by

$$
t^{2}-C_{N_{c}}\left(v, u_{k}\right) t+\Lambda_{N=2}^{2 N_{c}-N_{f}} \prod_{i=1}^{N_{f}}\left(v+m_{i}\right)=0,
$$

where $C_{N_{c}}$ is a degree $N_{c}$ polynomial in $v, C_{N_{c}}=v^{N_{c}}+\cdots$, with coefficients that depends on the moduli $u_{k}$, and $m_{i}\left(i=1, \ldots, N_{f}\right)$ are the quark masses.

\subsection{The D6-Branes}

To describe the Higgs branch of the theory, it is useful to introduce the D6-branes in the system, as we have seen in the Type IIA set-up. In Mtheory, the D6-branes are Kaluza-Klein Monopoles described by a TaubNUT space [40]. One of the complex structures of this Taub-NUT space is the same as one of the complex structures of the ALE space of $A_{n-1}$-type:

$$
y x=\Lambda_{N=2}^{2 N_{c}-N_{f}} \prod_{i=1}^{N_{f}}\left(v+m_{i}\right) .
$$




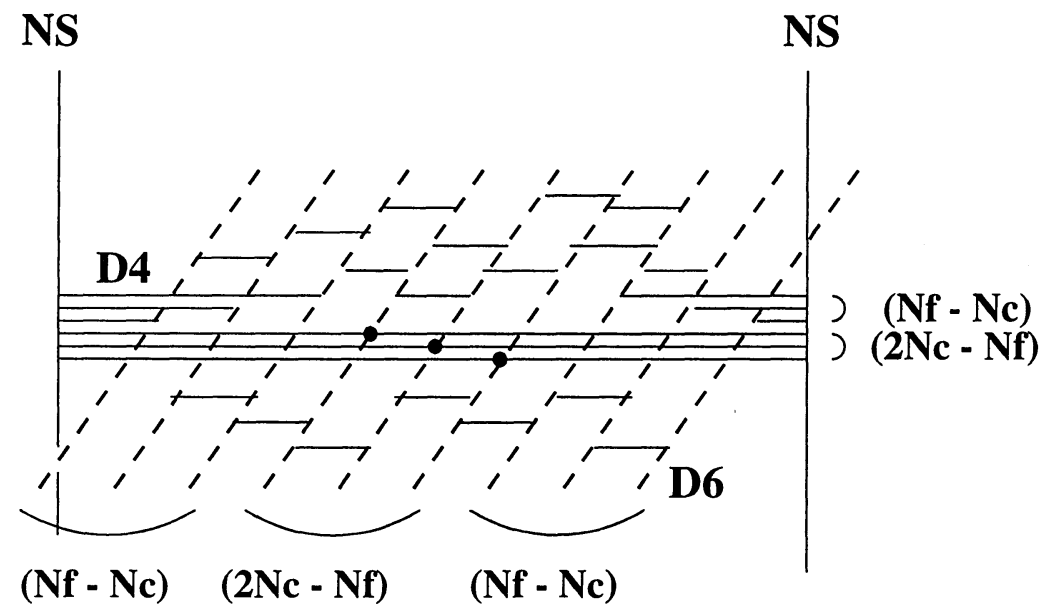

Figure 3: The baryonic branch in the type IIA picture.

The D6-branes are located at $x=y=0, v=-m_{i}$. In this framework, the Riemann surface $\Sigma$ is defined as a curve in this surface given by

$$
y+x=C_{N_{c}}\left(v, u_{k}\right) .
$$

It is easy to see that this description is the same as (3.2) under the identification $y=t$.

The Type IIA brane configuration is invariant under the rotations in the $x^{4}, x^{5}$ and $x^{7}, x^{8}, x^{9}$ directions, which we denote $U(1)_{4,5}$ and $S U(2)_{7,8,9}$ if the order parameters are also rotated appropriately. These are interpreted as the classical $U(1)$ and $S U(2) R$-symmetry groups of the four-dimensional theory on the brane worldvolume. In the M-theory configuration, $S U(2)_{7,8,9}$ is preserved but $U(1)_{4,5}$ is broken. We can preserve the discrete subgroup $\mathbb{Z}_{4 N_{c}-2 N_{f}}{ }^{4}$ of $U(1)_{4,5}$ if we modify the $U(1)_{4,5}$ action so that the variables $x$ and $y$ have charge $2 N_{c}$. The full $U(1)$ symmetry is restored if we assign the instanton charge $\left(4 N_{c}-2 N_{f}\right)$ to the factor $\Lambda_{N=2}^{2 N_{c}-N_{f}}$, reflecting the axial anomaly of the $U(1)_{R}$. We list here the modified $U(1)_{4,5}$ charges

$$
\begin{array}{cccc}
x & y & v & \Lambda_{N=2}^{2 N_{c}-N_{f}} \\
2 N_{c} & 2 N_{c} & 2 & 4 N_{c}-2 N_{f} .
\end{array}
$$

When some $m_{i}$ coincide, corresponding D6-branes are located at the same position in the $x^{4}, x^{5}$ directions, but they can be separated in the $x^{6}$ direction [5]. When $n$ of the bare masses are the same, the surface

\footnotetext{
${ }^{4}$ Since the $\mathbb{Z}_{2}$ subgroup acts trivially on the space-time coordinates and on the order parameters, we often call this a discrete $\mathbb{Z}_{2 N_{c}-N_{f}}$ R-symmetry.
} 
(3.3) develops an $A_{n-1}$ singularity. The separation of the D6-branes in the $x^{6}$ direction corresponds to the resolution of this singularity [10]. This resolution makes it possible to identify the Higgs branch of the $N=2$ theory on the fivebrane worldvolume. We now digress to give a brief description of the resolution of the $A_{n-1}$ singularity (see [41] for more detailed discussion).

Resolution of $A_{n-1}$ Singularity

The complex surface

$$
y x=v^{n}
$$

embedded in the $x-y-v$ space has a singularity at the origin. The resolution of this means a smooth complex surface that is mapped onto this singular surface in such a way that the map is an isomorphism except on the inverse image of the singular point. This is explicitly given as follows.

The resolved surface is covered by $n$ complex planes $U_{1}, U_{2}, U_{3}, \ldots, U_{n}$ with coordinates $\left(y_{1}=y, x_{1}\right),\left(y_{2}, x_{2}\right), \ldots,\left(y_{n}, x_{n}=y\right)$ which are mapped to the singular $A_{n-1}$ surface by

$$
U_{i} \ni\left(y_{i}, x_{i}\right) \longmapsto\left\{\begin{array}{l}
y=y_{i}^{i} x_{i}^{i-1} \\
x=y_{i}^{n-i} x_{i}^{n+1-i} \\
v=y_{i} x_{i} .
\end{array}\right.
$$

The planes $U_{i}$ are glued together by $x_{i} y_{i+1}=1$ and $y_{i} x_{i}=y_{i+1} x_{i+1}$. The map onto the singular $A_{n-1}$ surface is an isomorphism except on the inverse image of the singular point $x=y=v=0$. The inverse image consists of $n-1 \mathbb{C P}^{1} \mathrm{~s} C_{1}, C_{2}, \ldots, C_{n-1}$ where $C_{i}$ is the locus of $y_{i}=0$ in $U_{i}$ and $x_{i+1}=0$ in $U_{i+1}$, and is coordinatized by $x_{i}$ and $y_{i+1}$ that are related by $x_{i} y_{i+1}=1 . C_{i}$ and $C_{j}$ do not intersect unless $j=i \pm 1$, and $C_{i-1}$ and $C_{i}$ intersect transversely at $y_{i}=x_{i}=0$.

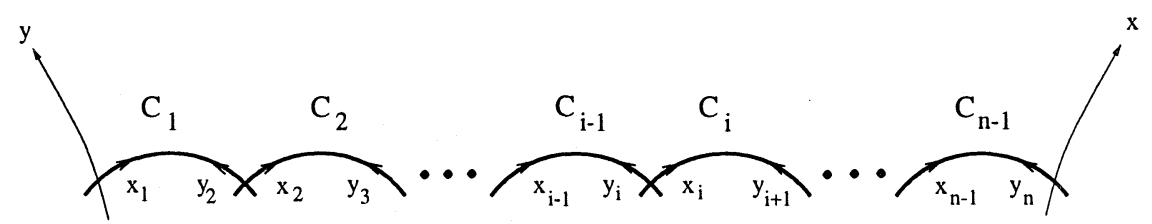

Figure 4: Resolution of $A_{n-1}$ singularity.

In what follows in this section, we turn off the bare mass: $m_{i}=0$ for any $i$. We separate the D6-branes in the $x^{6}$ direction and the eleven-dimensional space-time is then given by $\mathbb{R}^{7}$ times the resolved $A_{N_{f}-1}$ surface. In this case, the fivebrane is still described by the same equation (3.4) where $x, y, v$ are now considered as the functions (3.7) with $n=N_{f}$. The position of 
the D6-branes are interpreted as the $N_{f}$ intersection points of the rational curves $C_{1}, C_{2}, \ldots, C_{N_{f}-1}$.

\subsection{The Higgs Branch}

In the Type IIA picture, the Higgs branch is described by D4-branes suspended between $\mathrm{D} 6$-branes where they can move in the $x^{7}, x^{8}, x^{9}$ directions. Likewise, in the present context, the transition to the Higgs branch occurs when the fivebrane intersects with the D6-branes. This is possible only when the image (3.4) in the $x-y-v$ space of the curve passes through the singular point $x=y=v=0$. Thus, we must have $C_{N_{c}}(v=0)=0$, or in other words $C_{N_{c}}(v)$ factorizes as

$$
C_{N_{c}}(v)=v^{r}\left(v^{N_{c}-r}+u_{2} v^{N_{c}-r-2}+\cdots+u_{N_{c}-r}\right),
$$

where $r>0$.

Now, we describe what this curve looks like in the resolved $A_{N_{f}-1}$ surface. We first consider the case in which the coefficients $u_{2}, \ldots, u_{N_{c}-r}$ are generic. In particular $u_{N_{c}-r} \neq 0$.

It is convenient to look at the curve by separating it into two pieces; one is near, the other is away from the singularity $x=y=v=0$. Away from the singular point $x=y=v=0$, we can consider the curve as embedded in the original $x-y-v$ space because there is no distinction from the resolved surface in this region. As can be seen by looking at the two equations (3.3)-(3.4), $v$ never vanishes in this region of the curve. Thus, we can safely divide the coordinates $x$ and $y$ by some power of $v$. If $2 r \leq n$ they can be divided by $v^{r}$, while they can be divided by $v^{\left[N_{f} / 2\right]}$ if $2 r>N_{f}$. Then, we see that this piece of the curve is equivalent with the generic curve for the $S U\left(N_{c}-r\right)$ gauge theory with $\left(N_{f}-2 r\right)$ flavors and thus has genus $\left(N_{c}-r-1\right)$ for $2 r \leq N_{f}$, while it is some special genus $\left(N_{c}-\left[N_{f} / 2\right]-1\right)$ curve of the $S U\left(N_{c}-\left[N_{f} / 2\right]\right)$ gauge theory with $\left(N_{f}-2\left[N_{f} / 2\right]\right)$ flavors if $2 r>N_{f}$.

Near $x=y=v=0$, however, we must recall that we are actually considering the resolved $A_{N_{f}-1}$ surface. Thus, we must describe the curve in the $N_{f}$ patches as described above. Before doing this, it is useful to remark that the higher order terms $v^{r+1}, v^{r+2}, \ldots$ are negligible near $v=0$ compared to $v^{r}$. Thus, nothing essential is lost if we replace the defining equation $y+x=v^{r}\left(u_{N_{c}-r}+\cdots\right)=0$ by $y+x=v^{r}$. On the $i$-th patch $U_{i}$, the equation looks like

$$
y_{i}^{i} x_{i}^{i-1}+y_{i}^{N_{f}-i} x_{i}^{N_{f}+1-i}=y_{i}^{r} x_{i}^{r} .
$$

If $N_{f} \geq 2 r$, one of the three terms is of lowest order both in $y_{i}$ and $x_{i}$ : For $1 \leq i \leq r$, the lowest order term is the first term on the LHS, for 
$r+1 \leq i \leq N_{f}-r$, it is the term on the RHS, and for $N_{f}-r+1 \leq i \leq N_{f}$ it is the second term on the LHS. Thus, the equation factorizes as

$$
\begin{aligned}
& y_{i}^{i} x_{i}^{i-1}\left(1+y_{i}^{N_{f}-2 i} x_{i}^{N_{f}-2 i+2}-y_{i}^{r-i} x_{i}^{r+1-i}\right)=0 \\
& i=1, \ldots, r \\
& y_{i}^{r} x_{i}^{r}\left(y_{i}^{i-r} x_{i}^{i-r-1}+y_{i}^{N_{f}-r-i} x_{i}^{N_{f}+1-r-i}-1\right)=0 \\
& i=r+1, \ldots, N_{f}-r \\
& y_{i}^{N_{f}-i} x_{i}^{N_{f}+1-i}\left(y_{i}^{2 i-N_{f}} x_{i}^{2 i-N_{f}-2}+1-y_{i}^{i-N_{f}+r} x_{i}^{i-N_{f}+r-1}\right)=0 \\
& i=N_{f}-r+1, \ldots, N_{f} .
\end{aligned}
$$

We see that the curve consists of several components. One component, which we call $C$, is the zero of the last factor of the above equations. This extends to the one in the region away from $x=y=v=0$ which we have already considered. The other components are the rational curves $C_{1}, \ldots, C_{N_{f}-1}$. Recall that $C_{i}$ is defined by $y_{i}=0$ in $U_{i}$ and $x_{i+1}=0$ in $U_{i+1}$. In general, these have multiplicities. As is evident from the above factorized form, the component $C_{i}$ has multiplicity $\ell_{i}$ where $\ell_{i}=i$ for $i=1, \ldots, r, \ell_{i}=r$ for $i=r+1, \ldots, N_{f}-r$, and $\ell_{i}=N_{f}-i$ for $N_{f}-r+1, \ldots, N_{f}-1$. Note that the component $C$ intersects with $C_{r}$ and $C_{N_{f}-r}$ as we can see by looking at the equation at $i=r, r+1$ and $i=N_{f}-r, N_{f}-r+1$. The curve is depicted in figure 5 .

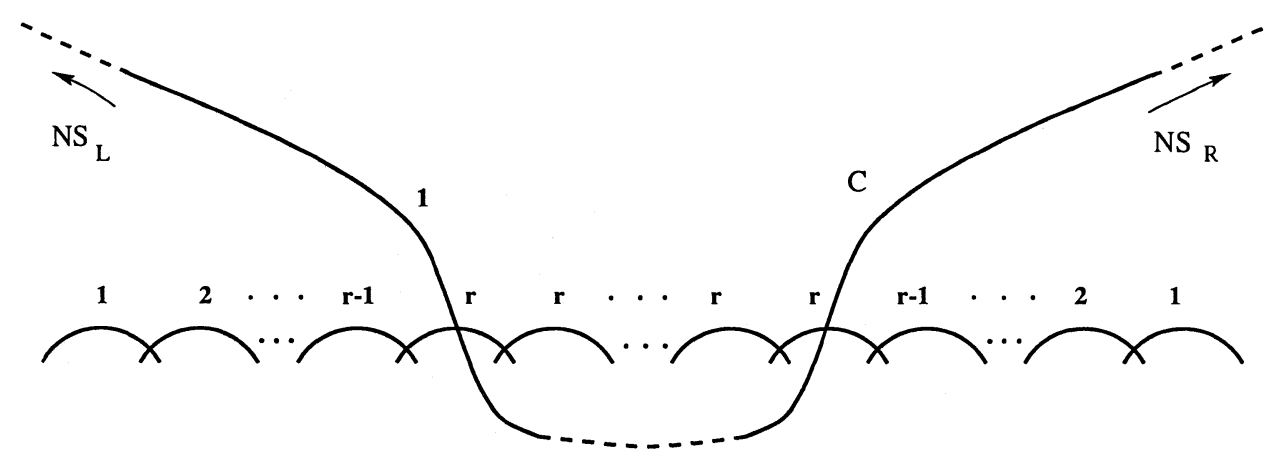

Figure 5: Non-baryonic branch root in M-Theory.

If $2 r>N_{f}$, the structure of degeneration of the curve is the same as the case $r=\left[N_{f} / 2\right]$. Recalling the behavior away from $x=y=v=0$, we conclude that the cases $2 r>N_{f}$ can be considered as some special cases of $r=\left[N_{f} / 2\right]$.

As noted in [10], once the curve degenerates and $\mathbb{C P}^{1}$ components are generated, they can move in the $x^{7}, x^{8}, x^{9}$ directions. This motion together with the integration of the chiral two-forms on such $\mathbb{C P}^{1}$ 's parametrize the 
Higgs branch of the four-dimensional theory. Since the $\mathbb{C P}^{1}$ components are $\ell_{i} C_{i}, i=1, \ldots, N_{f}-1$, the quaternionic dimension of the $r$-th Higgs branch is

$$
\sum_{i=1}^{N_{f}-1} \ell_{i}=2 \sum_{i=1}^{r-1} i+\left(N_{f}-2 r+1\right) \times r=r\left(N_{f}-r\right) .
$$

In view of the fact that there are $\left(N_{c}-r-1\right)$ parameters to deform the infinite component $C$ in the $x-y-v$ direction, we can identify this as the $r$-th non-baryonic branch emanating from an $\left(N_{c}-r-1\right)$-dimensional subvariety of the Coulomb branch, as introduced in [38].

If we look at figure 5 , it is evident how to identify the corresponding configuration in the Type IIA picture. The $\mathbb{C P}^{1}$ components $\ell_{i} C_{i}$ correspond to the $\ell_{i} \mathrm{D} 4$-branes stretched between the $i$-th and $i+1$-th D6-branes. That these are the only allowed configurations of D4-branes gives a proof of the $s$ rule, conjectured in [5], which forbids more than one D4-brane to be stretched between a NS 5-brane and a D6-brane. Further discussion on the $s$-rule will be given in sections 5 and 6 .

\section{The Baryonic Branch}

For the case $N_{f} \geq N_{c}$, in addition to the non-baryonic branches, there is a baryonic branch in which the gauge group is completely Higgsed [38]. It has quaternionic dimension $N_{f} N_{c}-\left(N_{c}^{2}-1\right)$ and it emanates from a point in the Coulomb branch. Here we look at the curve at the baryonic branch root, and see how the transition to the baryonic branch is possible.

One of the basic property of the baryonic branch root is that it is invariant under the discrete $R$-symmetry group $\mathbb{Z}_{2 N_{c}-N_{f}}$. This requires all the color Casimirs $u_{k}$ to be vanishing ${ }^{5}$. In this case $C_{N_{c}}(v)=v^{N_{c}}+v^{N_{f}-N_{c}}$. Thus, it is one of the non-baryonic branch roots with $r_{*}=N_{f}-N_{c}$ (note that $\left.N_{f}-2 r_{*}=2 N_{c}-N_{f} \geq 0\right)$. The equation $y+x=v^{N_{c}}+v^{N_{f}-N_{c}}$ then factorizes as

$$
\left(y-v^{N_{c}}\right)\left(y-v^{N_{f}-N_{c}}\right) / y=0 .
$$

Namely, the infinite curve $C$ factorizes into two rational curves $-C_{L}$ and $C_{R}$ corresponding to $y=v^{N_{c}}$ and $y=v^{N_{f}-N_{c}}$ respectively.

The dimension of the baryonic branch is greater than that of the $r_{*}$-th non-baryonic branch by 1 (in quaternionic dimension). The former is $N_{f} N_{c}-$ $\left(N_{c}^{2}-1\right)$ while the latter is $r_{*}\left(N_{f}-r_{*}\right)=N_{f} N_{c}-N_{c}^{2}$. This difference corresponds to the missing dimension of the baryonic branch in the type IIA picture. How can we account for this difference? We note that the two curves $C_{L}$ and $C_{R}$ intersects at $\left(2 N_{c}-N_{f}\right)$ points. This follows from the defining equations $y=v^{N_{c}}$ and $y=v^{N_{f}-N_{c}}$ and the fact that they never intersect near

\footnotetext{
${ }^{5}$ According to the convention of [38] where $C_{N_{c}}(v)=\prod_{a=1}^{N_{c}}\left(v-\phi_{a}\right)$, the root of the baryonic branch is at $\phi=\left(0, \ldots, 0, \omega, \ldots, \omega^{2 N_{c}-N_{f}}\right)$ where $\omega=e^{2 \pi i /\left(2 N_{c}-N_{f}\right)}$.
} 


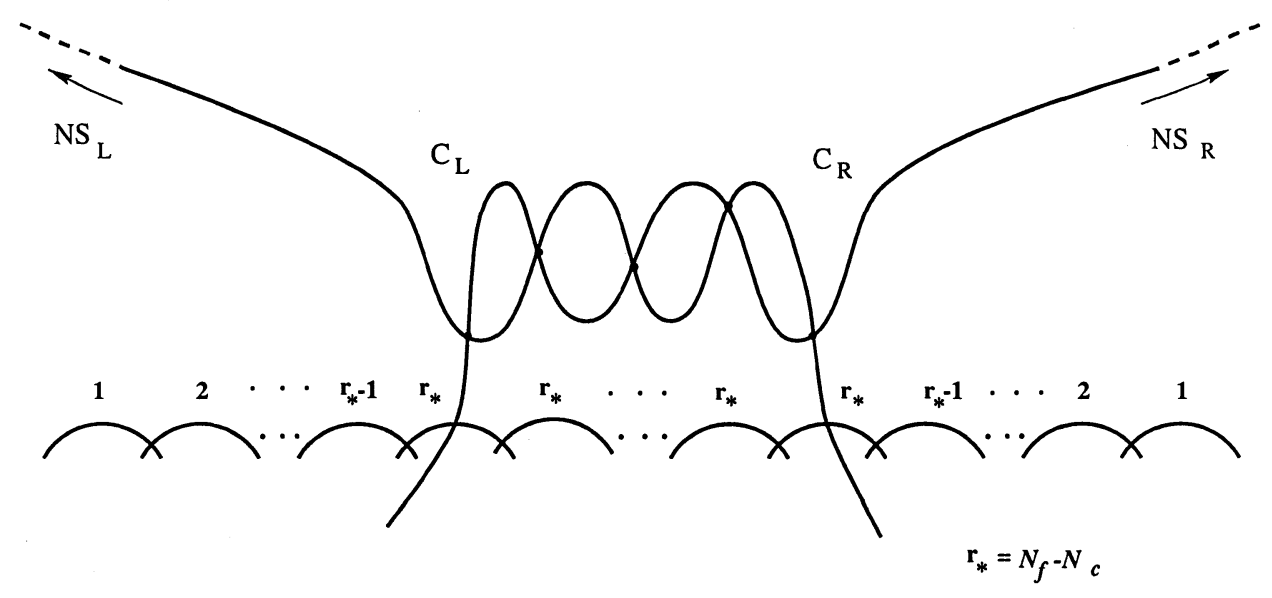

Figure 6: The baryonic branch root in M-Theory.

$x=y=v=0$. On the other hand, the infinite curve $C$ at the generic point of the $r_{*}$-th non-baryonic branch root has genus $N_{c}-r_{*}-1=2 N_{c}-N_{f}-1$. This means that, as the curve approaches the baryonic branch root, it degenerates at $\left(2 N_{c}-N_{f}\right)$ points and factorizes into two rational curves. Thus the curve $C$ at the baryonic branch root (or equivalently the union of $C_{L}$ and $C_{R}$ ) describes the abelian gauge theory with gauge group $U(1)^{2 N_{c}-N_{f}-1}=$ $\prod_{i}^{2 N_{c}-N_{f}-1} U(1)_{i}$. There are $\left(2 N_{c}-N_{f}\right)$ massless electrons with charges $(-1,0, \ldots, 0),(1,-1,0, \ldots, 0), \ldots,(0, \ldots, 1,-1)$, and $(0, \ldots, 0,1)$ coming from the degeneration points on the curve (the charges can be read off from the intersection relations of the vanishing cycles). It is easy to see that such a theory has one-dimensional Higgs branch. In this way, we have identified the missing +1 of the dimension from the M-theory point of view.

\section{Rotating the Brane Configuration}

By adding a mass term to the adjoint chiral multiplet in the $N=2$ vector multiplet, the $N=2$ supersymmetry is broken to $N=1$. In this section, we study the corresponding configuration of fivebranes in M-theory. In the Type IIA picture, this corresponds to changing the relative orientation of the NS 5-branes.

Since one of the 5 -branes is going to be extended in the $\left(x^{8}, x^{9}\right)$ directions also, there is only $N=1$ supersymmetry left on $\mathbb{R}^{1,3}$. To describe the corresponding configuration of the M-theory fivebrane, let us introduce a complex coordinate

$$
w=x^{8}+i x^{9}
$$




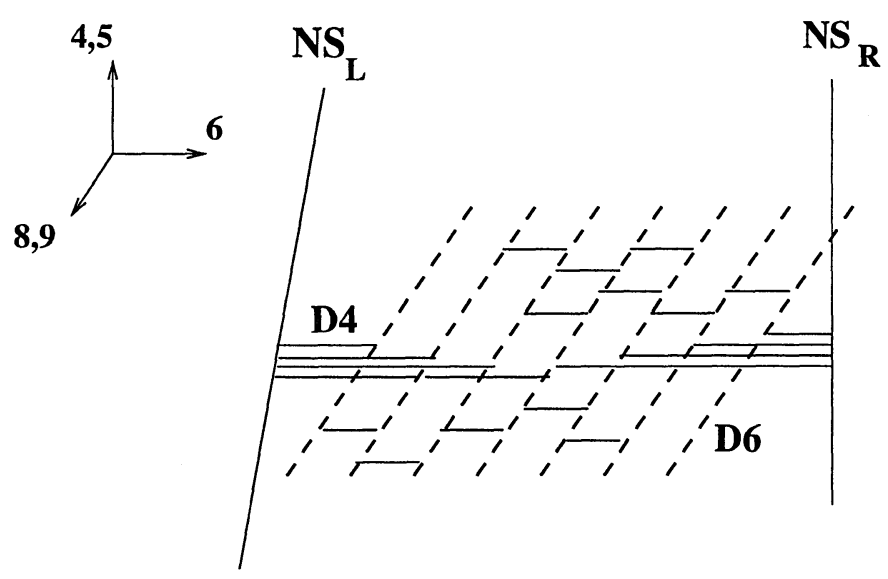

Figure 7: Rotating the left NS 5-brane.

Before breaking the $N=2$ supersymmetry, the fivebrane is located at $w=0$. We then rotate the left NS 5-brane toward the $w$ direction while keeping the right NS 5-brane intact. Since the two NS 5-branes correspond to the two asymptotic regions with $v \rightarrow \infty$ where $t=y \sim v^{N_{c}}$ and $x \sim v^{N_{c}}$ respectively where the latter is equivalent to $t \sim \Lambda_{N=2}^{2 N_{c}-N_{f}} v^{N_{f}-N_{c}}$, the rotation means that we impose the boundary condition as

$$
\begin{aligned}
& w \rightarrow \mu v \quad \text { as } v \rightarrow \infty, t \sim v^{N_{c}} \\
& w \rightarrow 0 \quad \text { as } v \rightarrow \infty, t \sim \Lambda_{N=2}^{2 N_{c}-N_{f}} v^{N_{f}-N_{c}} .
\end{aligned}
$$

We can identify $\mu$ as the mass of the adjoint chiral multiplet by using the $R$-symmetries. Recall that the $N=2$ configuration is invariant under the rotation groups $U(1)_{4,5}$ and $S U(2)_{7,8,9}$ corresponding to the $R$-symmetry of the field theory on the brane worldvolume, where the action of $U(1)_{4,5}$ is modified as (3.5). After the rotation, $S U(2)_{7,8,9}$ is broken to $U(1)_{8,9}$ if the parameter $\mu$ in (4.2) is assigned the $U(1)_{4,5} \times U(1)_{8,9}$ charge $(-2,2)$. Since this is the same as the $R$-charges of the mass of the adjoint field and since there is no other parameter charged with respect to $U(1)_{8,9}$, the two quantities should be identified. We list below the charges of the coordinates and parameters.

$\begin{array}{ccc} & U(1)_{4,5} & U(1)_{8,9} \\ v & 2 & 0 \\ w & 0 & 2 \\ y=t & 2 N_{c} & 0 \\ x & 2 N_{c} & 0 \\ \mu & -2 & 2 \\ \Lambda_{N=2}^{2 N_{c}-N_{f}} & 4 N_{c}-2 N_{f} & 0\end{array}$


As a preliminary remark, recall that in general the curve consists of several components at the Higgs branch root. To rotate the curve in such a case, we pick the component, called $C$, that extends to infinity in the $v$ direction and rotate that component. If the curve is not at the Higgs branch root, the curve to be rotated is $\Sigma$ itself, but we denote it as $C$ also in this case. At the baryonic branch root, the component $C$ further factorizes into two rational curves $C_{L}$ and $C_{R}$. In this case, the rotation is actually much easier than the other cases, but we shall give a separate discussion in view of its importance in a later section.

We should not expect to be able to rotate the brane configuration (3.2) for arbitrary values of $u_{k}$ 's. This is obvious from the field theory point of view since the adjoint mass lifts the Coulomb branch of the $N=2$ theory and the $u_{k}$ 's are drawn to roots of Higgs branches. It is also clear from the classical brane picture. If the D4-branes suspended between the NS 5branes are apart, the two NS 5-branes have to remain parallel (if we force the NS 5-branes to change their relative orientations, the D4-branes get twisted and the supersymmetry is completely broken). In the M-theory picture, it is possible to rotate a brane only when all the handles of the curve $C$ degenerate and $C$ can be considered as a single cylinder with some points pairwise attached. This can be seen by the following argument.

Suppose a curve $C$ is rotatable. First, we note that the projection of the rotated curve $\widetilde{C}$ on the $t-v$ plane remains the same as (3.2). This follows from the conservation of the $U(1)_{8,9}$ symmetry. When $\mu$ is small, $w$ on $\widetilde{C}$ can be expressed as a function of $v$ and $t$. Thus the projection of $\widetilde{C}$ on the $t-v$ plane can still be described by some equation in $(t, v)$. To see that this equation is the same as (3.2), we note that $\mu$ is the only parameter that carries the $U(1)_{8,9}$ charge. Therefore we cannot deform the equation (3.2) without breaking the $U(1)_{8,9}$ symmetry. This means that the rotated curve $\widetilde{C}$ can be considered as the graph of the "function" $w$ of the original curve $C$. In order to clarify the property of this function $w$, we compactify the curve $C$. We note that both of the two asymptotic regions $-v \rightarrow \infty, t \sim v^{N_{c}}$ and $v \rightarrow \infty, t \sim v^{N_{f}-N_{c}}\left({ }^{6}\right)-$ are well parametrized by the coordinate $v$. Thus, we can compactify the curve by attaching the two points at infinity $1 / v=0$, obtaining a compact Riemann surface $\bar{C}$. The asymptotic condition (4.2) is equivalent to saying that $w$ is a meromorphic function of $\bar{C}$ which has only a simple pole at one point (one of the points at infinity). Such a function exists only when the curve $\bar{C}$ is equivalent to $\mathbb{C P}^{1}$.

This completes the proof that the rotatable curve $C$ should be completely degenerate (i.e. all the $\alpha$-cycles vanish). The proof based on the asymptotic condition (4.2) also shows that the rotated curve $\widetilde{C}$ is a cylinder which is globally parametrized by the coordinate $w$. The two points at infinity of the

\footnotetext{
${ }^{6}$ We put $\Lambda_{N=2}=1$ for a while for simplicity.
} 


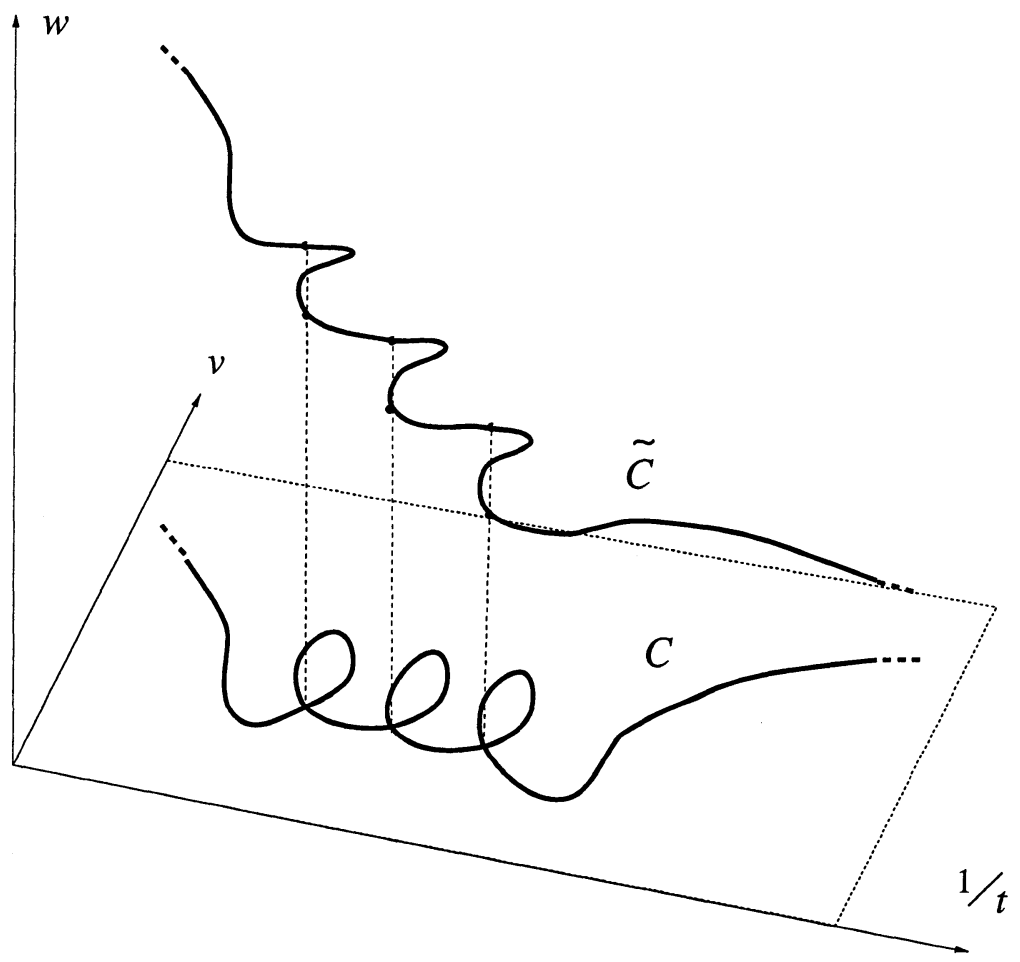

Figure 8: Rotated curve as a graph of $w$.

curve correspond to $w=0$ and $w=\infty$.

Thus, we can express $t$ and $v$ in terms of $w$ by rational functions:

$$
v=P(w), \quad t=Q(w) .
$$

Since $v$ and $t$ never diverge except at the infinity $w=0, \infty$, these rational functions are polynomials in $w$ up to a factor of some power of $w: P(w)=$ $w^{a} p(w), Q(w)=w^{b} q(w)$ where $a$ and $b$ are some (possibly negative) integers and $p(w)$ and $q(w)$ are polynomials of $w$ which we may assume non-vanishing at $w=0$. Near one of the points at infinity $w=\infty, v$ and $t$ behave as $v \sim \mu^{-1} w$ and $t \sim v^{N_{c}}$ by (4.2). Thus, the rational functions are of the form $P(w)=w^{a}\left(w^{1-a}+\cdots\right) / \mu$ and $Q(w)=\mu^{-N_{c}} w^{b}\left(w^{N_{c}-b}+\cdots\right)$. Let us look at the other infinity $w=0$ and consider the cylinder to be compactified at this point. A neighborhood of this point is well parametrized by $1 / v$ which takes the value zero at $w=0$. Recall that $w$ is the global coordinate of the cylinder and hence extends to the global coordinate of the compactified cylinder. Namely, $1 / v$ and $w$ are two good coordinates that vanish at the same point. Thus they must be linearly related $w \sim$ const $/ v$ in the limit 
$w \rightarrow 0$. The function $P(w)$ then is of the form

$$
P(w)=\frac{w^{2}+\cdots}{\mu w}=\frac{\left(w-w_{+}\right)\left(w-w_{-}\right)}{\mu w} .
$$

Since $t \sim v^{N_{f}-N_{c}}$ and $w \sim$ const $/ v$ as $w \rightarrow 0$, we obtain $b=N_{c}-N_{f}$ and thus $Q(w)=\mu^{-N_{c}} w^{N_{c}-N_{f}}\left(w^{N_{f}}+\cdots\right)$. For $N_{f}>0$, by the equation $y x=v^{N_{f}}$ defining the space-time, $t=0$ (i.e. $y=0$ ) implies $v=0$. Therefore the zeros of the polynomial $w^{N_{f}}+\cdots$ coincide with the zeros $w_{+}$and $w_{-}$of $P(w)$. This way, we have determined the form of $Q(w)$ also:

$$
Q(w)=\mu^{-N_{c}} w^{N_{c}-N_{f}}\left(w-w_{+}\right)^{r}\left(w-w_{-}\right)^{N_{f}-r}
$$

for some $r=0,1, \cdots,\left[N_{f} / 2\right]$. We have imposed the bound $r \leq\left[N_{f} / 2\right]$ because the reflection $r \leftrightarrow N_{f}-r$ is compensated by the exchange $w_{+} \leftrightarrow w_{-}$.

For $r>0$, both $v$ and $t$ get small near $w=w_{ \pm}$and behave as $t \sim v^{r}$ and $t \sim v^{N_{f}-r}$. This is the property of the curve at the $r$-th baryonic branch root, as can be seen by looking at the relation (3.2) between $v$ and $t$ near $t=v=0$ which is approximately $t^{2}-v^{r} t+v^{N_{f}}=0$. To see this in another way, we note that the component $C$ at the $r$-th non-baryonic branch root intersects the exceptional curve $x=y=v=0$ at two points near which $y=t$ and $v$ behave as $t \sim v^{r}$ and $t \sim v^{N_{f}-r}$. Thus, we conclude that the $r>0$ curve given by (4.5) and (4.6) is the rotation of the component $C$ at one of the $r$-th non-baryonic branch roots. For $r=0$, there is a point $\left(w=w_{+}\right)$at which $v=0$ but $t \neq 0$. In this case the function $C_{N_{c}}(v)$ does not vanish at $v=0$, which means that the curve does not pass through the D6-branes in $x=y=v=0$. This is possible only if the curve is not at a Higgs branch root.

The values $w_{ \pm}$are determined by the condition that $v=P(w)$ and $t=Q(w)$ with (4.5)-(4.6) satisfy identically the relation $t+v^{N_{f}} / t=C_{N_{c}}(v)=$ $v^{r} C_{N_{c}-r}(v)$. This reads as

$$
\begin{gathered}
w^{2 N_{c}-N_{f}}\left(w-w_{-}\right)^{N_{f}-2 r}+\mu^{2 N_{c}-N_{f}}\left(w-w_{+}\right)^{N_{f}-2 r}= \\
=\left(w-w_{+}\right)^{N_{c}-r}\left(w-w_{-}\right)^{N_{c}-r}+u_{2}(\mu w)^{2}\left(w-w_{+}\right)^{N_{c}-2-r}\left(w-w_{-}\right)^{N_{c}-2-r}+ \\
+\cdots+u_{N_{c}-r}(\mu w)^{N_{c}-r}
\end{gathered}
$$

up to the factor $\left(w-w_{+}\right)^{r}\left(w-w_{-}\right)^{r} /(\mu w)^{N_{c}}$. The matching of the subleading and the lowest order terms in powers of $w$ requires (recovering $\Lambda_{N=2}$ )

$$
\begin{aligned}
& \left(1-\frac{r}{N_{c}}\right) w_{+}+\left(1-\frac{N_{f}-r}{N_{c}}\right) w_{-}=0, \\
& \left(w_{+}\right)^{N_{c}-N_{f}+r}\left(w_{-}\right)^{N_{c}-r}=(-1)^{N_{f}}\left(\mu \Lambda_{N=2}\right)^{2 N_{c}-N_{f}}
\end{aligned}
$$


and these determine $w_{+}$and $w_{-}$up to a $\mathbb{Z}_{2 N_{c}-N_{f}}$ phase rotation. Matching of other terms determines the coefficients $u_{2}, \ldots, u_{N_{c}-r}$, and these are uniquely expressed in terms of $w_{ \pm}$so that the $\mathbb{Z}_{2 N_{c}-N_{f}}$ action on $w_{ \pm}$leads to the natural action on $u_{i}$. Note that in the case $r=N_{f} / 2\left(N_{f}\right.$ even), the action of the $\mathbb{Z}_{2}$ subgroup is identified with the exchange of $w_{+}$and $w_{-}$which does nothing on the rotated curve.

Remark on the $r=r_{*}$ case: If $N_{f} \geq N_{c}$, there is a subtlety concerning the case in which $r$ is $r_{*}=N_{f}-N_{c}$. For this value of $r$, the equation (4.7) implies $w_{+}=0$. That is, the curve is given by $v=\left(w-w_{-}\right) / \mu, t=\left(w-w_{-}\right)^{N_{c}} / \mu^{N_{c}}$ and one of the asymptotic regions $v \rightarrow \infty, t \sim v^{N_{f}-N_{c}}$ is absent. This means that such a curve cannot be realized as a curve of the form (3.2). Thus, we must exclude this case from the list of rotated curves. Actually, this is one of the components of the rotated curve at the baryonic branch which we now describe.

\section{The Baryonic Branch}

The rotation of the curve at the baryonic branch root is straightforward. The component $C$ at the baryonic branch factorizes into two pieces $-C_{L}$ described by $t=v^{N_{c}}, w=0$ and $C_{R}$ described by $t=v^{N_{f}-N_{c}}, w=0$. The rotation can be done just by replacing $w=0$ for $C_{L}$ by $w=\mu v$. The curve is explicitly given by

$$
\widetilde{C}_{L}\left\{\begin{array} { l } 
{ t = v ^ { N _ { c } } } \\
{ w = \mu v }
\end{array} \quad C _ { R } \left\{\begin{array}{l}
t=\Lambda_{N=2}^{2 N_{c}-N_{f}} v^{N_{f}-N_{c}} \\
w=0
\end{array}\right.\right.
$$

\section{Summary}

To summarize, we have identified all possible curves that can be rotated. The curve at the baryonic branch can be rotated, and the result is given by (4.9). Some curves at the non-baryonic branch roots and some curves away from the Higgs branch roots are also rotatable. The result of the rotation of these curves is given by the equations $\left(r=0,1, \ldots,\left[N_{f} / 2\right], r \neq r_{*}\right)$

$$
\begin{aligned}
v & =\frac{\left(w-w_{+}\right)\left(w-w_{-}\right)}{\mu w}, \\
t & =\mu^{-N_{c}} w^{N_{c}-N_{f}}\left(w-w_{+}\right)^{r}\left(w-w_{-}\right)^{N_{f}-r},
\end{aligned}
$$

where $w_{ \pm}$are determined by (4.7) and (4.8). For each $r<N_{f} / 2, r \neq r_{*}$, there are $\left(2 N_{c}-N_{f}\right)$ solutions related by the $\mathbb{Z}_{2 N_{c}-N_{f}}$ action, while for $r=N_{f} / 2\left(N_{f}\right.$ even $)$ there are $\left(N_{c}-N_{f} / 2\right)$ solutions. Before the rotation, the curve for $r>0, r \neq r_{*}$ is at the $r$-th non-baryonic branch root, while the curve for $r=0$ is not at a Higgs branch root. 
We can perform a similar analysis in the case in which the quark mass term $m_{f} \sum_{i} \widetilde{Q}^{i} Q_{i}$ is turned on. For generic values of $m_{f}$, there is no baryonic branch and the curve $C$ never factorizes. We only have to repeat the same procedure for the unfactorized curves by replacing $v$ by $v+m_{f}$, and the result is a slight modification of (4.10)-(4.11) with (4.7)-(4.8). That is, we replace (4.10) and (4.7) by

$$
\begin{aligned}
& v+m_{f}=\frac{\left(w-w_{+}\right)\left(w-w_{-}\right)}{\mu w}, \\
& \left(1-\frac{r}{N_{c}}\right) w_{+}+\left(1-\frac{N_{f}-r}{N_{c}}\right) w_{-}+\mu m_{f}=0 .
\end{aligned}
$$

For every $r=0,1, \ldots,\left[N_{f} / 2\right]$, there are $2 N_{c}-N_{f}$ solutions ${ }^{7}$.

\section{Comparison with Field Theory}

The fivebrane configuration we found here encodes various information on the moduli space of vacua of the $N=1$ gauge theory. Let us compare it with the results of the field theory analysis in section 2 . Here we will restrict our attention to the regions of the moduli space where the vev of the baryon fields $B, \tilde{B}$ vanish, and postpone the discussion of non-zero vev for $\tilde{B} B$ to section 6 . We will find that the brane provides us with an exact description of the moduli space of vacua.

\section{Interpretation of $w_{ \pm}$}

Let us discuss the meaning of the values of $w_{ \pm}$. In the Type IIA set-up in which all the D6-branes are sent to the infinity $x^{6}=+\infty$, there are $N_{f}$ semi-infinite D4-branes ending on the right NS 5-brane from the right. The fact that $t=v=0$ at $w=w_{ \pm}$means that $w=w_{ \pm}$are the asymptotic positions in the $w=x^{8}+i x^{9}$ direction of these semi-infinite D4-branes. Moreover, the order of zero in (4.11) says that $r$ of the $N_{f}$ D4-branes are at $w=w_{+}$in the limit $x^{6} \rightarrow+\infty$ and the remaining $N_{f}-r$ are at $w=w_{-}$.

A $U\left(N_{f}\right)$ symmetry is associated with these semi-infinite D4-branes. From the point of view of the four-dimensional field theory on the D4-branes which are finite in the $x^{6}$ direction, this appears as the global symmetry. When the D4-branes are separated from each other, the global symmetry is broken. If the separation is in the $v=x^{4}+i x^{5}$ direction, it is interpreted as the explicit breaking due to the bare mass $\left(m_{f}\right)_{i}$ of the quarks, since these are the only parameters charged under $U(1)_{4,5}$ that can break the $U\left(N_{f}\right)$ flavor symmetry. If the separation is in the $w$ direction, it must be interpreted

\footnotetext{
${ }^{7}$ For special values of $m_{f}$, there are $r$ such that $w_{+}$or $w_{-}$vanishes. These presumably correspond to the vacua with meson matrix having Jordan blocks which is mentioned in a footnote in section 2 .
} 
as due to a quantity with $U(1)_{8,9}$ charge 2 that can break the $U\left(N_{f}\right)$ flavor symmetry. The only such quantity is the meson vev $M_{j}^{i}=\widetilde{Q}^{i} Q_{j}$. Thus, we interpret the position in the $w$ direction of the semi-infinite D4-branes as the eigenvalues of the meson matrix $M_{j}^{i}$.

This interpretation can be verified as follows. First, the fact that there are at most two values $w_{ \pm}$for the asymptotic $w$ position of the $N_{f}$ D4-branes is consistent with the field theory result that there are at most two eigenvalues $m_{(1)}$ and $m_{(2)}$ of the meson matrix. Moreover, for each degeneracy type $r$, the positions $w_{+}$and $w_{-}$of degeneracy $r$ and $N_{f}-r$ agree with the eigenvalues $m_{(1)}$ and $m_{(2)}$ of degeneracy $r$ and $N_{f}-r$ respectively, up to an overall phase that depends only on $N_{c}$.

Let us compare these two quantities in more detail.

$0<N_{f}<N_{c}$

Consider first the massless case. The extrema of the exact superpotential (2.7) were found to satisfy equations (2.17) and (2.18) and solved by (2.19). The solution for $r=0$ in (2.16) is a special case of (2.19). The equations defining $w_{ \pm}$(4.7) and (4.8) are identical to (2.17) and (2.18) up to an overall phase factor; thus we have

$$
w_{+}=(-1)^{\frac{N_{c}}{2 N_{c}-N_{f}}} m_{(1)}, \quad w_{-}=(-1)^{\frac{N_{c}}{2 N_{c}-N_{f}}} m_{(2)} .
$$

Given the interpretation of $w_{ \pm}$as the eigenvalues of the meson matrix, we see that the fivebrane in M-theory describes correctly the moduli space of vacua on the $N=1$ gauge theory which is obtained in field theory as the extrema of (2.7), including the dynamical generation of the Affleck-DineSeiberg superpotential and the non-renormalization of (2.7).

Consider next the massive case. The extrema of the exact superpotential (2.20) satisfy equations (2.21) and (2.22). The equations defining $w_{ \pm}(4.13)$ and (4.8) are, as in the massless case, identical to (2.21) and (2.22) up to an overall phase factor and $w_{ \pm}$are related to $m_{(1)}, m_{(2)}$ by $(4.14)$. We see that also in the massive case the fivebrane in M-theory describes correctly the exact moduli space of vacua on the $N=1$ gauge theory.

$N_{f}=N_{c}$

The extrema of the superpotential (2.25) were found to satisfy equations (2.30) and (2.31) and solved by (2.33). The equations defining $w_{ \pm}(4.7)$ and (4.8) are identical to (2.30) and (2.31) up to an overall phase factor and $w_{ \pm}$are related to $m_{(1)}, m_{(2)}$ by (4.14). In this case we do not have a field theory proof that the superpotential (2.25) is exact. Since we expect the M-theory fivebrane to describe the exact moduli space of vacua, this result may be regarded as an evidence for the non-renormalization theorem of (2.25). In the limit $\mu \rightarrow \infty$, the low energy theory becomes $N=1$ SQCD 
with $N_{f}=N_{c}$. In this case the moduli space of vacua is modified quantum mechanically and the singularity at the origin is resolved. Indeed, the brane captures this phenomenon as we shall see below.

$\underline{N_{c}+1 \leq N_{f}}$

In section 2 we used the magnetic description in order to find the vacua.

The extrema of the superpotential (2.35) satisfy equations (2.17) and (2.18), which are also the equations defining $w_{ \pm}(4.7)$ and (4.8), again up to an overall phase factor. $w_{ \pm}$are related to $m_{(1)}, m_{(2)}$ by $(4.14)$ as in the previous cases. In this case we also do not have a field theory proof that the superpotential (2.35) is exact. Our result here using the M-theory fivebrane suggests that it is.

To summarize: With the interpretation of $w_{ \pm}$as the eigenvalues of the meson matrix, what we showed is that the fivebrane in M-theory describes the exact moduli space of vacua and captures the quantum phenomena such as the dynamical generation of the Affleck-Dine-Seiberg superpotential for $N_{f}<N_{c}$ and the quantum modification to the classical moduli space of vacua when $N_{f}=N_{c}$. Moreover it provided us with non-renormalization theorems for superpotentials which have not been proven by field theory methods.

\section{The $\mu \rightarrow \infty$ Limit: $N=1$ SQCD}

In the last section, we have found the configuration of the fivebrane for a finite value of the adjoint mass $\mu$. In this section, we take the $\mu \rightarrow \infty$ limit of this configuration and compare it with the known result of the $N=1$ supersymmetric field theory.

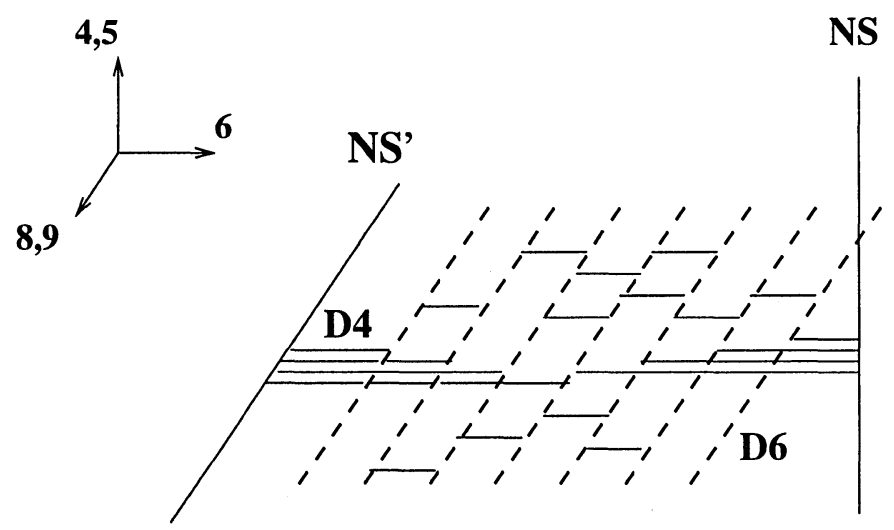

Figure 9: The $\mu \rightarrow \infty$ limit of type IIA configuration. 
Recall that we have been considering rotation of the left NS 5-brane which corresponds to the asymptotic region $t \sim v^{N_{c}}, v \rightarrow \infty$. After rotation, this region behaves as $w \rightarrow \infty, v \sim \mu^{-1} w$, and

$$
t \sim \mu^{-N_{c}} w^{N_{c}} .
$$

Since the $N_{c}$ D4-branes end on the left NS 5-brane also in the right angle limit, we expect the relation $t \sim w^{N_{c}}$ to hold in the limit $\mu \rightarrow \infty$ as well. For this, it is evident from (5.1) that we must rescale $t$ by a factor $\mu^{N_{c}}$ and introduce the rescaled variable

$$
\widetilde{t}=\mu^{N_{c}} t
$$

Since the space-time is asymptotically a flat cylinder with flat coordinate $x^{6}+i x^{10}=R \log (1 / t)$, this rescaling simply corresponds to the shift of the origin in the $x^{6}, x^{10}$ directions. Moreover, the rescaling fits nicely with the symmetry property and the renormalization group flow of the corresponding four-dimensional physics, as we now see.

Recall that we are considering the eleven-dimensional space-time to be a flat $\mathbb{R}^{7}$ times the Taub-NUT space described by (3.3) where $y$ is identified with $t$. Using the rescaled variable (5.2) or equivalently $\widetilde{y}=\mu^{N_{c}} y$, the space-time is described by

$$
\widetilde{y} x=\mu^{N_{c}} \Lambda_{N=2}^{2 N_{c}-N_{f}} \prod_{i=1}^{N_{f}}\left(v+m_{i}\right) .
$$

This expression has a smooth limit as $\mu \rightarrow \infty$ provided the constant $\Lambda_{N=1}$ given by

$$
\Lambda_{N=1}^{3 N_{c}-N_{f}}=\mu^{N_{c}} \Lambda_{N=2}^{2 N_{c}-N_{f}}
$$

is kept finite. It may appear that this choice of limit is ambiguous because we could rescale the coordinate $x$ also. However, this is not allowed since the fivebrane behaves near the other infinity $w \rightarrow 0$ as $x \sim v^{N_{c}}, v \rightarrow \infty$, and this should also be preserved in the $\mu \rightarrow \infty$ limit. The relation (5.4) is the same as the renormalization group matching condition of the corresponding four-dimensional field theory. This space-time together with the fivebrane in it which we are going to describe is invariant under the rotation groups $U(1)_{4,5}$ and $U(1)_{8,9}$ where the charges of the new parameters and coordinates are given by

$$
\begin{array}{ccc} 
& U(1)_{4,5} & U(1)_{8,9} \\
v & 2 & 0 \\
w & 0 & 2 \\
\widetilde{y}=\widetilde{t} & 0 & 2 N_{c} \\
x & 2 N_{c} & 0 \\
\Lambda_{N=1}^{3 N_{c}-N_{f}} & 2 N_{c}-2 N_{f} & 2 N_{c} \\
\mu & -2 & 2
\end{array} .
$$


In particular, the charges of the factor $\Lambda_{N=1}^{3 N_{c}-N_{f}}$ mean that the groups $U(1)_{4,5}$ and $U(1)_{8,9}$ are broken to their discrete subgroups $\mathbb{Z}_{2 N_{c}-2 N_{f}}$ and $\mathbb{Z}_{2 N_{c}}$ respectively. This dictates precisely the anomaly of the corresponding $U(1)$ $R$-symmetry groups.

\section{1 $S U\left(N_{c}\right)$ Without Matter}

In this case, the curve describing the $N=2$ Coulomb branch is given by

$$
t^{2}-C_{N_{c}}\left(v, u_{k}\right) t+\Lambda_{N=2}^{2 N_{c}}=0 .
$$

There are $N_{c}$ points on the Coulomb branch where the curve is completely degenerate, and these points are related to each other by the unbroken discrete $\mathbb{Z}_{4 N_{c}}$ subgroup of $U(1)_{4,5}$ acting as $v \rightarrow v \mathrm{e}^{\frac{2 \pi i}{2 N_{c}}}, t \rightarrow-t$ which has the effect $\Lambda_{N=2}^{2} \rightarrow \Lambda_{N=2}^{2} \mathrm{e}^{\frac{2 \pi i}{N_{c}}}$ on the curve.

At one of these points, the curve takes the form,

$$
v=\Lambda_{N=2}^{2} t^{-1 / N_{c}}+t^{1 / N_{c}},
$$

and its rotation is given by

$$
\begin{aligned}
v & =\mu \Lambda_{N=2}^{2} w^{-1}+\mu^{-1} w \\
t & =\mu^{-N_{c}} w^{N_{c}} .
\end{aligned}
$$

Before taking the $\mu \rightarrow \infty$ limit, we rescale $t$ as $\tilde{t}=\mu^{N_{c}} t$ (5.2). The equations (5.7) and (5.8) can then be rewritten as

$$
\begin{aligned}
v & =\Lambda_{N=1}^{3} \widetilde{t}^{-1 / N_{c}}+\mu^{-1} \widetilde{t}^{1 / N_{c}}, \\
w & =\widetilde{t}^{1 / N_{c}} \\
v w & =\Lambda_{N=1}^{3}+\mu^{-1} w^{2}
\end{aligned}
$$

where, following (5.4)

$$
\Lambda_{N=1}^{3}=\mu \Lambda_{N=2}^{2} .
$$

This curve has a smooth $\mu \rightarrow \infty$ limit if we send $\Lambda_{N=2} \rightarrow 0$ at the same time so that $\Lambda_{N=1}$ remains finite. Dropping the terms multiplied by $\mu^{-1}$, the curve in this limit becomes

$$
\begin{aligned}
v & =\Lambda_{N=1}^{3} \tilde{t}^{-1 / N_{c}}, \\
w & =\widetilde{t}^{1 / N_{c}}, \\
v w & =\Lambda_{N=1}^{3} .
\end{aligned}
$$

We note that $\Lambda_{N=1}$ characterizes the size of the fivebrane configuration (5.12). The $\mathbb{Z}_{2 N_{c}}$ subgroups of $U(1)_{4,5}$ and $U(1)_{8,9}$ have the same effect

$$
\Lambda_{N=1}^{3} \rightarrow \mathrm{e}^{\frac{2 \pi i}{N_{c}}} \Lambda_{N=1}^{3}
$$


on the curve. The $N_{c}$ curves, or fivebranes, are related by this discrete $\mathbb{Z}_{2 N_{c}}$ symmetry group which is spontaneously broken to $\mathbb{Z}_{2}$. This is what we have observed in the four-dimensional $N=1$ super-Yang-Mills theory.

\section{2 $S U\left(N_{c}\right)$ With $N_{f}$ Massless Matter}

\subsubsection{Non-Baryonic Branches}

In terms of the rescaled variables, the equations describing the rotated brane at the root of the $r$-th non-baryonic branch $\left(r=0,1, . .,\left[N_{f} / N_{c}\right]\right.$; $\left.r \neq N_{f}-N_{c}\right)$ are

$$
\begin{aligned}
& \mu^{-N_{c}} \tilde{t}^{2}-v^{r} C_{N_{c}-r}\left(v, u_{k}\right) \tilde{t}+\Lambda_{N=1}^{3 N_{c}-N_{f}} v^{N_{f}}=0 \\
& \tilde{t}=w^{N_{c}-N_{f}}\left(w-w_{+}\right)^{r}\left(w-w_{-}\right)^{N_{f}-r} \\
& v w=\mu^{-1}\left(w-w_{+}\right)\left(w-w_{-}\right)
\end{aligned}
$$

where $w_{ \pm}$are determined by (4.7) and (4.8). In particular, $w_{ \pm}$are of the form

$$
w_{ \pm}=c_{ \pm} \mu \Lambda_{N=2},
$$

where $c_{ \pm}$are non-zero numerical constants depending on $N_{c}, N_{f}$ and $r$. We recall that $\Lambda_{N=2}$ and $\Lambda_{N=1}$ are related by (5.4) and we send $\Lambda_{N=2}$ to zero so that $\Lambda_{N=1}$ is kept finite. Since the order parameters $u_{k}$ are powers of $\Lambda_{N=2}$ and are independent of $\mu$, they vanish in the $\mu \rightarrow \infty$ limit, which means that $v^{r} C_{N_{c}-r}(v) \rightarrow v^{N_{c}}$. Thus, the equation (5.14) has the smooth limit described by

$$
\tilde{t}=\Lambda_{N=1}^{3 N_{c}-N_{f}} v^{N_{f}-N_{c}} .
$$

How the other two equations (5.15) and (5.16) behave in this limit depends on the behavior of $w_{ \pm} \sim \mu \Lambda_{N=2}$. Since

$$
\mu \Lambda_{N=2}=\left(\mu^{N_{c}-N_{f}} \Lambda_{N=1}^{3 N_{c}-N_{f}}\right)^{\frac{1}{2 N_{c}-N_{f}}}
$$

we should distinguish three cases, $N_{f}<N_{c}, N_{f}=N_{c}$ and $N_{f}>N_{c}$.

When $N_{f}<N_{c}, \mu \Lambda_{N=2}$ diverges in the limit $\mu \rightarrow \infty$. This means that the curve defined by (5.14), (5.15) and (5.16) becomes infinitely elongated along the $x^{6}$ direction. Thus the $\mu \rightarrow \infty$ limit of the brane configuration does not provide a field theory in four dimensions. This is consistent with the field theory result that there is no supersymmetric vacuum for the corresponding $N=1$ theory.

When $N_{f}>N_{c}, \mu \Lambda_{N=2}$ vanishes for $\mu \rightarrow \infty$. In this limit, the equations (5.14)-(5.16) become

$$
v^{N_{c} \tilde{t}}=\Lambda_{N=1}^{3 N_{c}-N_{f}} v^{N_{f}}
$$




$$
\begin{aligned}
\tilde{t} & =w^{N_{c}}, \\
v w & =0 .
\end{aligned}
$$

It may appear that only $\tilde{t}=w^{N_{c}}$ and $v=0$ is allowed since the second equation seems to imply that $w=0$ means $\tilde{t}=0$. However, the double limit $w \rightarrow 0, \mu \rightarrow \infty$ of the equation (5.15) is subtle. As we will see later, $\tilde{t} \neq 0$, $w=0$ is allowed. Thus, the correct interpretation of these equations is that the curve splits into two components in this limit:

$$
C_{L}\left\{\begin{array} { l } 
{ \tilde { t } = w ^ { N _ { c } } , } \\
{ v = 0 , }
\end{array} \quad C _ { R } \left\{\begin{array}{l}
\tilde{t}=\Lambda_{N=1}^{3 N_{c}-N_{f}} v^{N_{f}-N_{c}}, \\
w=0 .
\end{array}\right.\right.
$$

The component $C_{L}$ corresponds to the $\mathrm{NS}^{\prime}$ 5-brane and the D4-branes attached to it, while the component $C_{R}$ corresponds to the NS 5-brane and the attached D4-branes. It is interesting to note that all the non-baryonic branch roots have the same limit.

To be more precise, there are other components corresponding to D4branes stretched between the D6-branes. These are rational curves located at the exceptional divisor $x=y=v=0$. In addition to the ones which are present already before rotation (i.e. $N=2$ limit $\mu=0$ ), there are also rational curves which appear only in the $\mu \rightarrow \infty$ limit. We will describe how they appear shortly. It turns out that all the non-baryonic branch roots have the same limit as well even if we take these rational curves into account.

When $N_{f}=N_{c}, \mu \Lambda_{N=1}$ is equal to $\Lambda_{N=1}^{2}$. In the limit $\mu \rightarrow \infty$ the equations (5.14)-(5.16) become

$$
\begin{aligned}
\tilde{t} & =\Lambda_{N=1}^{2 N_{c}} \\
\tilde{t} & =\left(w-c_{+} \Lambda_{N=1}^{2}\right)^{r}\left(w-c_{-} \Lambda_{N=1}^{2}\right)^{N_{c}-r}, \\
v w & =0 .
\end{aligned}
$$

As in the case of $N_{f}>N_{c}$, the correct interpretation of these is that the curve splits into two components.

$$
C_{L}\left\{\begin{array} { l } 
{ \tilde { t } = ( w - c _ { + } \Lambda _ { N = 1 } ^ { 2 } ) ^ { r } ( w - c _ { - } \Lambda _ { N = 1 } ^ { 2 } ) ^ { N _ { c } - r } , } \\
{ v = 0 , }
\end{array} C _ { R } \left\{\begin{array}{l}
\tilde{t}=\Lambda_{N=1}^{2 N_{c}} \\
w=0
\end{array}\right.\right.
$$

Unlike the case of $N_{f}>N_{c}$, the final configuration of the curve depends on $r$, i.e. different non-baryonic branch roots go to different limits. As in the $N_{f}>N_{c}$ case, there are other finite components. As we will see, those which appear only in the $\mu \rightarrow \infty$ limit are located at $w=w_{ \pm}$.

\subsubsection{Baryonic Branch}

The baryonic branch exists for $N_{f} \geq N_{c}$. Before the right angle limit, the rotated curve is given by (4.9). In terms of the rescaled variables, it is written 
as

$$
\widetilde{C}_{L}\left\{\begin{array} { l } 
{ \tilde { t } = w ^ { N _ { c } } } \\
{ v = \mu ^ { - 1 } w }
\end{array} \quad C _ { R } \left\{\begin{array}{l}
\tilde{t}=\Lambda_{N=1}^{3 N_{c}-N_{f}} v^{N_{f}-N_{c}} \\
w=0 .
\end{array}\right.\right.
$$

In the limit $\mu \rightarrow \infty$, these become

$$
C_{L}\left\{\begin{array} { l } 
{ \tilde { t } = w ^ { N _ { c } } } \\
{ v = 0 }
\end{array} \quad C _ { R } \left\{\begin{array}{l}
\tilde{t}=\Lambda_{N=1}^{3 N_{c}-N_{f}} v^{N_{f}-N_{c}} \\
w=0
\end{array}\right.\right.
$$

For $N_{f}>N_{c}$, the baryonic branch root has the same limit as the nonbaryonic roots given by (5.21). Thus in this case, all the roots of the Higgs branches converge to the same curve. On the other hand, for $N_{f}=N_{c}$, (5.25) are different from (5.23) for the non-baryonic branches. This reflects the fact that the quantum moduli space for the $N=1$ theory with $N_{f}=N_{c}$ is different from the classical one while the moduli space for $N_{f}>N_{c}$ does not receive quantum corrections. This point will be discussed further in the next section.

\section{3 $S U\left(N_{c}\right)$ With Massive Matter}

If $N_{f}<N_{c}$ and the quarks are massless, the $\mu \rightarrow \infty$ limit of the theory has no supersymmetric vacua as we have just seen by brane analysis and also in Section 2 from the point of view of field theory.

However we can stabilize the vacuum by adding a quark mass term which breaks $U(1)_{4,5}$ but preserves $U(1)_{8,9}$. From the field theory analysis, we have seen that the non-baryonic branch with only $r=0$ survives in this case. So let us examine how it looks like from the point of view of the brane configuration.

If all the quarks have the same mass $m_{f}$, the curve for finite $\mu$ is

$$
\begin{aligned}
v+m_{f} & =\frac{\left(w-w_{+}\right)\left(w-w_{-}\right)}{\mu w}, \\
\mu^{N_{c}} t & =w^{N_{c}-N_{f}}\left(w-w_{-}\right)^{N_{f}} .
\end{aligned}
$$

In the limit $\mu \rightarrow \infty$,

$$
\begin{aligned}
& w_{+} \simeq-\mu m_{f} \\
& w_{-} \simeq\left(\frac{\Lambda_{N=1}^{3 N_{c}-N_{f}}}{m_{f}^{N_{c}-N_{f}}}\right)^{1 / N_{c}}
\end{aligned}
$$

Therefore the curve in this limit becomes

$$
v=\left(m_{f}^{N_{f}} \Lambda_{N=1}^{3 N_{c}-N_{f}}\right)^{1 / N_{c}} w^{-1}
$$




$$
\tilde{t}=w^{N_{c}-N_{f}}\left(w-\left(\frac{\Lambda_{N=1}^{3 N_{c}-N_{f}}}{m_{f}^{N_{c}-N_{f}}}\right)^{1 / N_{c}}\right)^{N_{f}},
$$

where $\tilde{t}=\mu^{N_{c}} t$ as before. The limit $\mu \rightarrow \infty$ has been taken so that $\Lambda_{N=1}$ given in (5.4) remains finite. Note that there are $N_{c}$ solutions related to each other by the action of the discrete $\mathbb{Z}_{2 N_{c}}$ subgroup of $U(1)_{8,9}$.

It is interesting to see how this configuration reduces to that for $N_{f}=0$ in the limit $m_{f} \rightarrow \infty$. If we define

$$
m_{f}^{N_{f}} \Lambda_{N=1}^{3 N_{c}-N_{f}}=\tilde{\Lambda}_{N=1}^{3 N_{c}}
$$

the curve (5.28) can be written as

$$
\begin{aligned}
v & =\frac{\tilde{\Lambda}_{N=1}^{3}}{w} \\
\tilde{t} & =w^{N_{c}-N_{f}}\left(w-\frac{\tilde{\Lambda}_{N=1}^{3}}{m_{f}}\right)^{N_{f}} .
\end{aligned}
$$

If we keep $\tilde{\Lambda}_{N=1}$ finite while we send $m_{f} \rightarrow \infty$, the space-time in this limit is just the flat cylinder given by

$$
\widetilde{y} x=\tilde{\Lambda}_{N=1}^{3 N_{c}},
$$

and the fivebrane (5.30) reduces to the pure Yang-Mills result (5.12). Once again, (5.29) is exactly the renormalization matching condition for the corresponding situation in the field theory.

On the other hand, the limit $m_{f} \rightarrow 0$ of (5.28) gives an infinitely elongated curve (the branch at $w=\left(\Lambda_{N=1}^{3 N_{c}-N_{f}} / m_{f}^{N_{c}-N_{f}}\right)^{1 / N_{c}}$ goes to the infinity). This corresponds to the fact that there is no supersymmetric vacuum for the $S U\left(N_{c}\right)$ theory with massless $N_{f}\left(<N_{c}\right)$ flavors.

\subsection{Generation of Rational Curves in the $\mu \rightarrow \infty$ Limit}

In the subsection 5.2.1, we mentioned that extra rational curves appear in the $\mu \rightarrow \infty$ limit. We will show this in this subsection. In the Type IIA picture, this corresponds to breaking the D4-branes attached to the NS' 5 -brane at the D6-branes, which becomes possible only in the right angle limit because of the $s$-rule. The appearance of such extra rational curves means a generation of extra flat directions of the $\mu=\infty$ theory. This will be important in the next section. We consider only the case $N_{f} \geq N_{c}$.

We recall that for finite angle rotation and $\mu<\infty$ the projection of the fivebrane in the Taub-NUT space (i.e. forgetting the $x^{0,1,2,3,7,8,9}$ direction) 
remains the same as the starting point $\mu=0$, provided we fix the $N=2$ scale $\Lambda_{N=2}$. In terms of the rescaled variable it is given by

$$
x+\mu^{-N_{c}} \widetilde{y}=v^{N_{c}}+\cdots .
$$

where $+\cdots$ are certain lower order terms in $v$. Here we stress that this equation describes the whole curve (projected onto the Taub-NUT space) including the rational curve components as well as the infinite component(s). The coefficients of the lower order terms $+\cdots$ in $v$ are given by positive powers of $\Lambda_{N=2}$, and hence are of negative powers in $\mu$ if $\Lambda_{N=1}$ is fixed. Thus, the $\mu \rightarrow \infty$ limit of the projection of the curve onto the Taub-NUT space is given by

$$
x=v^{N_{c}} .
$$

In principle, this could be different from the projection of the $\mu \rightarrow \infty$ curve because some components can go away to infinity in the $w$ direction. Later, we show that this does not happen in the case $N_{f} \geq N_{c}$ we are considering and indeed the $w$ values of any rational curve components are at finite values of $w$. As we will see, the $w$ values are actually zero except for the $N_{f}=N_{c}$ curves describing the non-baryonic branch root.

We first show how the projected curve (5.33) look like in the Taub-NUT space. Recall that the Taub-NUT space we are considering is the resolved $A_{N_{f}-1}$ surface. It is covered by $N_{f}$ patches with coordinates $\left(y_{1}, x_{1}\right), \ldots \ldots$, $\left(y_{N_{f}}, x_{N_{f}}\right)$ which are projected to the $\tilde{y}-x-v$ space by ${ }^{8}$

$$
\begin{aligned}
& \widetilde{y}=y_{i}^{i} x_{i}^{i-1}, \\
& x=y_{i}^{N_{f}-i} x_{i}^{N_{f}+1-i}, \\
& v=y_{i} x_{i},
\end{aligned}
$$

in which we have put $\Lambda_{N=1}=1$ for simplicity. The equation (5.33) looks in the $i$-th patch as

$$
y_{i}^{N_{f}-i} x_{i}^{N_{f}+1-i}=y_{i}^{N_{c}} x_{i}^{N_{c}} .
$$

In the first $N_{f}-N_{c}$ patches $\left(i=1, \ldots, N_{f}-N_{c}\right)$ the RHS is of lower order both in $y_{i}$ and $x_{i}$ than the LHS, while the LHS is of lower order than RHS in the remaining patches $\left(i=N_{f}-N_{c}+1, \ldots, N_{f}\right)$. Recall that the equations $y_{i}=x_{i+1}=0$ define a rational curve $C_{i}$. Thus, the curve includes as its components the rational curve $C_{i}$ with multiplicity $N_{c}$ for $1 \leq i \leq N_{f}-N_{c}$ and $N_{f}-i$ for $N_{f}-N_{c}+1 \leq i \leq N_{f}-1$. Namely, the multiplicities of

\footnotetext{
${ }^{8}$ We can obtain this from (3.7) in which we have put $\Lambda_{N=2}=1$ for simplicity: We first recover $\Lambda_{N=2}$ in (3.7) just by replacing the expression for $y$ by $y=\Lambda_{N=2}^{2 N_{c}-N_{f}} y_{i}^{i} x_{i}^{i-1}$. Then, by definition $\tilde{y}=\mu^{N_{c}} y=\Lambda_{N=1}^{3 N_{c}-N_{f}} y_{i}^{i} x_{i}^{i-1}$, obtaining (5.34).
} 
$C_{1}, \ldots, C_{N_{f}-1}$ are

$$
\underbrace{N_{c}, N_{c}, \ldots, N_{c}}_{N_{f}-N_{c}}, N_{c}-1, \ldots, 2,1 .
$$

In addition to these rational curve components, there are two components of infinite volume - the curve described by $x_{1}^{N_{c}}=0$ (i.e. $y_{1}$-axis with multiplicity $N_{c}$ ) in the first patch and the curve described by $y_{i}^{N_{f}-N_{c}-i} x_{i}^{N_{f}-N_{c}-i+1}=1$. As we will see, the actual curve projected to $x_{1}^{N_{c}}=0$ is an $N_{c}$-fold cover $C_{L}$ extending in the $w$ direction, while the curve $C_{R}$ projected to the latter is at $w=0$. If $N_{f}>N_{c}$, the projection of $C_{R}$ intersects with the rational curve $C_{N_{f}-N_{c}}$. In summary, the projected curve (5.33) is depicted in figure 10.
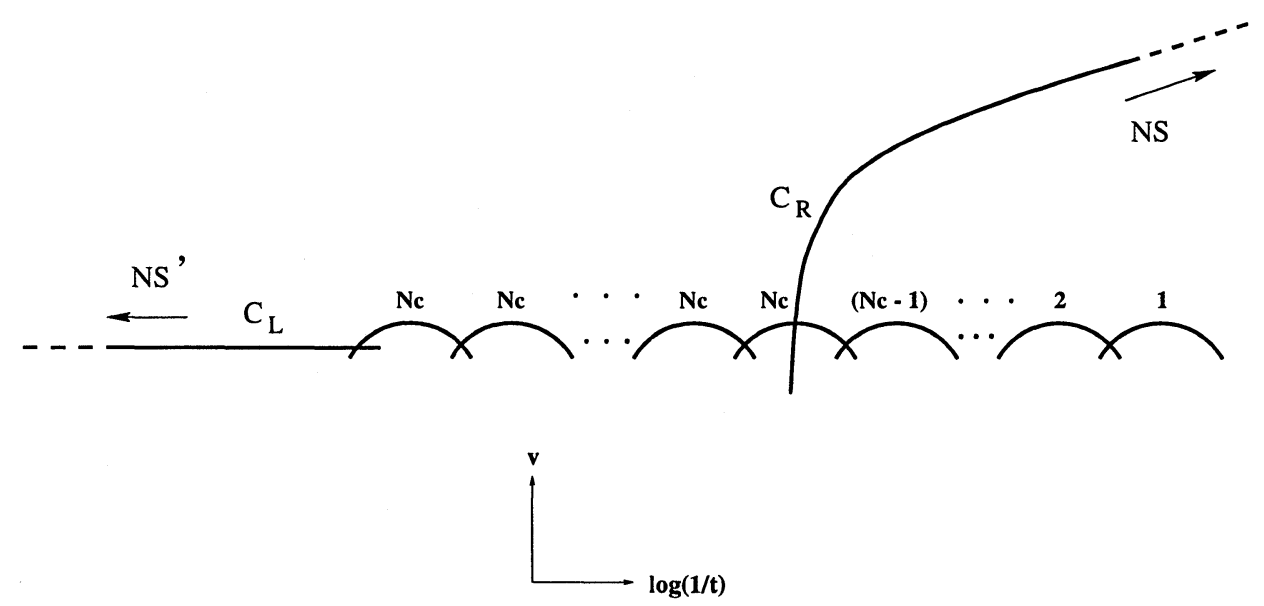

Figure 10: The projection (5.33) in the Taub-NUT space.

Note that the numbers of rational or infinite curve components have increased from any of the $N=2$ Higgs branch roots. This means that the infinite component of the finite $\mu$ curve degenerates in the $\mu \rightarrow \infty$ limit and has turned into a union of rational curve components and infinite component(s). We now exhibit this explicitly, proving that (5.33) is indeed the projection of the $\mu \rightarrow \infty$ curve.

\section{Baryonic Branch Root}

We recall that the curve of the baryonic branch root consists of $N_{f} N_{c}-N_{c}^{2}$ rational curve components and two infinite components $\widetilde{C}_{L}$ and $C_{R}$. The components other than $\widetilde{C}_{L}$ will stay the same after rotation as well as in the $\mu \rightarrow \infty$ limit. So we only have to consider the $\mu \rightarrow \infty$ limit of $\widetilde{C}_{L}$. The component $\widetilde{C}_{L}$ at finite $\mu$ given by (5.24) is described in the $i$-th patch by

$$
y_{i}=\mu^{i-1} w^{N_{c}-i+1},
$$




$$
x_{i}=\mu^{-i} w^{i-N_{c}} .
$$

Let us see this in the limit $\mu \rightarrow \infty$. If $i>N_{c}$, the first equation $y_{i} w^{i-N_{c}-1}=$ $\mu^{i-1}$ can never be satisfied for finite $y_{i}$ or $w$, and thus the curve disappears from this patch. So let us consider the case $i \leq N_{c}$. For $x_{i}$ to be finite, $x_{i} \sim 1, w$ must scale as $\mu^{i} w^{N_{c}-i} \sim 1$. Then, $y_{i}=\mu^{-1} w / x_{i} \sim \mu^{-1} w$ behaves as $y_{i}^{N_{c}-i} \sim \mu^{-\left(N_{c}-i\right)} w^{N_{c}-i} \sim \mu^{-N_{c}}$. This means that for every finite $x_{i}$, there are $N_{c}-i$ values of $y_{i}$ approaching to zero. Thus, we see that the limit includes the component $C_{i}$ with multiplicity $N_{c}-i$. If we repeat the same thing by interchanging $x_{i}$ and $y_{i}$, we see that the limit includes the component $C_{i-1}$ with multiplicity $N_{c}-i+1$. The location of these components are at $w=0$ because of the scaling relations $-\mu^{i} w^{N_{c}-i} \sim 1$ for $C_{i}$. In the first patch, there is another component $C_{L}$ in which $w$ is arbitrary:

$$
C_{L}\left\{\begin{array}{l}
y_{1}=w^{N_{c}} \\
x_{1}=0
\end{array}\right.
$$

In summary, the curve $\widetilde{C}_{L}$ degenerates in the $\mu \rightarrow \infty$ limit and consists of an infinite component $C_{L}$ and rational curves $C_{1}, C_{2}, \ldots, C_{N_{c}-1}$ of multiplicity $N_{c}-1, N_{c}-2, \ldots, 1$. Together with the components $C_{1}, C_{2}, \ldots, C_{N_{f}-1}$ and $C_{R}$ with multiplicity $1,2, \ldots, r_{*}-1, r_{*}, \ldots, r_{*}, r_{*}-1, \ldots, 2,1$ and 1 respectively (recall that $r_{*}=N_{f}-N_{c}$ ) which we have been omitting for a while, the total $\mu \rightarrow \infty$ curve consists of the components $C_{L}, C_{1}, C_{2}, \ldots, C_{r_{*}}, C_{r_{*}+1}$, $\ldots, C_{N_{f}-2}, C_{N_{f}-1}$ and $C_{R}$ with the multiplicities $1, N_{c}, N_{c}, \ldots, N_{c}, N_{c}-1$, $\ldots, 2,1$ and 1 respectively. This is depicted in figure 11 and figure 12 .

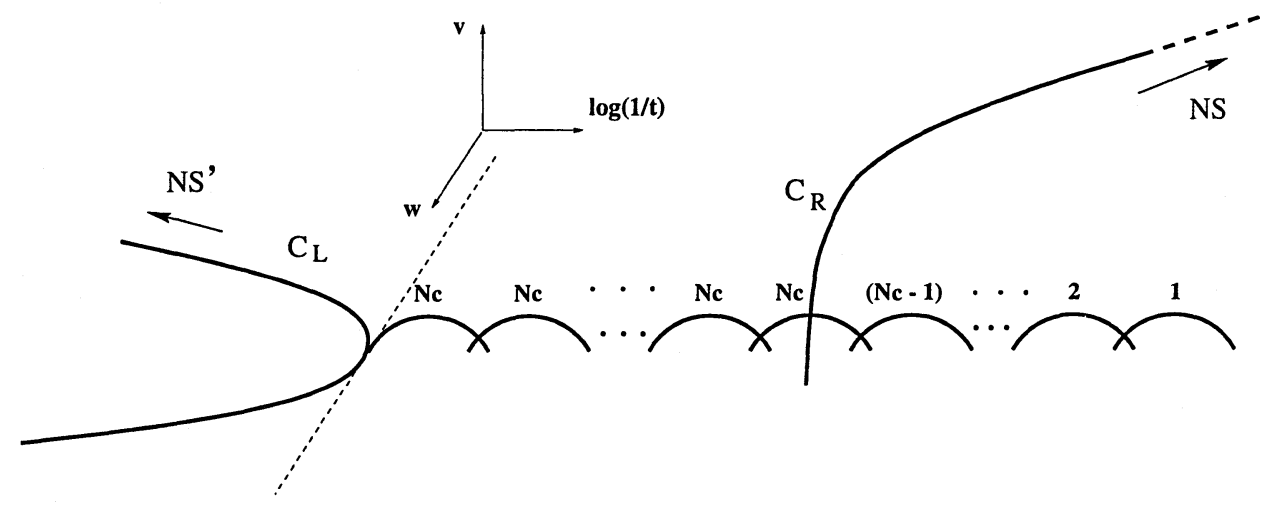

Figure 11: The $\mu \rightarrow \infty$ limit of the baryonic branch root $\left(N_{f}>N_{c}\right)$.

Non-Baryonic Branch Roots

$\underline{N_{f}=N_{c}}$ 


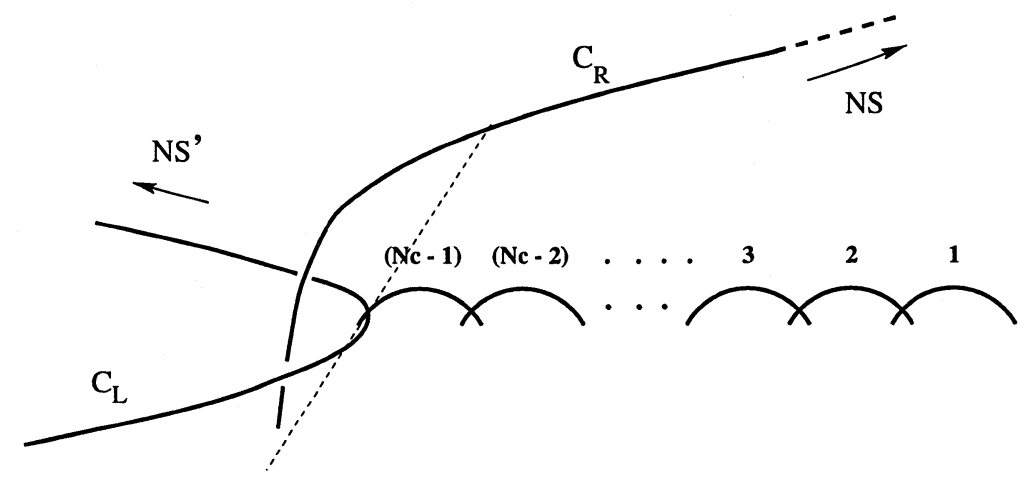

Figure 12: The $\mu \rightarrow \infty$ limit of the baryonic branch $\operatorname{root}\left(N_{f}=N_{c}\right)$.

Lets us look at the $\mu$ finite rotated curve of the $r$-th non-baryonic branch root of the $N_{f}=N_{c}$ case where $r=1, \ldots,\left[N_{c} / 2\right]$. The infinite component $C$ is described in the $i$-th patch by

$$
\begin{aligned}
& y_{i}=\mu^{i-1} w^{i-1}\left(w-w_{+}\right)^{r-i+1}\left(w-w_{-}\right)^{N_{f}-r-i+1} \\
& x_{i}=\mu^{-i} w^{-i}\left(w-w_{+}\right)^{i-r}\left(w-w_{-}\right)^{i-\left(N_{f}-r\right)} .
\end{aligned}
$$

Recall that for $N_{f}=N_{c}$ case, $w_{+}$and $w_{-}$have non-zero distinct limits as $\mu \rightarrow \infty$. There are three ways to scale $w$ in order to make $y_{i}$ and $x_{i}$ finite in the limit $\mu \rightarrow \infty$ :

(i) $w \rightarrow 0$ keeping $\mu w$ finite.

(ii) $w \rightarrow w_{+}$keeping $\mu^{i-\epsilon}\left(w-w_{+}\right)^{r-i+\epsilon}$ finite $(\epsilon=0,1)$.

(iii) $w \rightarrow w_{-}$keeping $\mu^{i-\epsilon}\left(w-w_{-}\right)^{N_{f}-r-i+\epsilon}$ finite $(\epsilon=0,1)$.

The scaling of type (i) is possible for every $i$, and the corresponding limit is an infinite curve $C_{R}$ given by $y_{i}^{i} x_{i}^{i-1}=1, w=0$. The scaling of type (ii) is possible only for $i \leq r$. For $\epsilon=0, x_{i}$ is finite and $y_{i}$ goes to zero as $y_{i}^{r-i} \sim \mu^{-r}$, while for $\epsilon=1, y_{i}$ is finite and $x_{i}$ goes to zero as $x_{i}^{r-i+1} \sim \mu^{-r}$. Thus, from the type (ii) limit, we obtain the components $C_{1}, C_{2}, \ldots, C_{r-1}$ at $w=w_{+}$with multiplicity $(r-1),(r-2), \ldots, 1$ respectively. The scaling of type (iii) is possible only for $i \leq N_{f}-r$. By the similar argument as in type (ii), one can see that we obtain the components $C_{1}, C_{2}, \ldots, C_{N_{f}-r-1}$ at $w=w_{-}$with multiplicity $\left(N_{f}-r-1\right),\left(N_{f}-r-2\right), \ldots, 1$ respectively. In the first patch $i=1$, there is another component $C_{L}$ given by $y_{1}=$ $\left(w-w_{+}\right)^{r}\left(w-w_{-}\right)^{N_{f}-r}, x_{1}=0$ which is infinite and parametrized by $w$. Together with the components which already exist before taking the limit, the $\mu \rightarrow \infty$ curve consists of the components depicted in figure 13 .

$\frac{N_{f}>N_{c}}{\text { Finally, we consider the } \mu \rightarrow \infty \text { limit of the } r \text {-th non-baryonic branch }}$ root, where $r=0,1, \ldots,\left[N_{f} / 2\right], r \neq r_{*}=N_{f}-N_{c}$. 


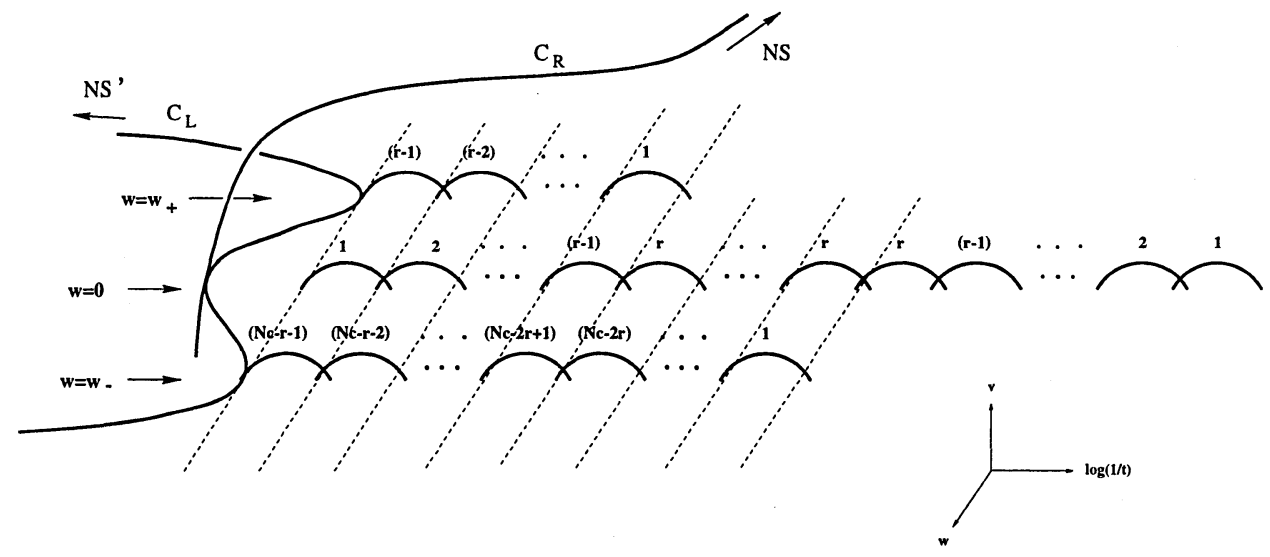

Figure 13: The $\mu \rightarrow \infty$ limit of the non-Baryonic branch root in the $N_{f}=N_{c}$ case.

In this case, both $w_{+}$and $w_{-}$vanish in the limit $\mu \rightarrow \infty$. More precisely, they scale as $w_{ \pm} \sim \mu^{-\beta}$ where $\beta=\left(N_{f}-N_{c}\right) /\left(2 N_{c}-N_{f}\right)$. There are three ways to scale $w$, keeping $y_{i}$ and $x_{i}$ to be finite in the limit:

(i) $w \sim \mu^{-\alpha}$ where $\alpha<\beta$.

(ii) $w \sim \mu^{-\alpha}$ where $\alpha>\beta$.

(iii) $w \sim w_{+}+\mu^{-\delta}$ where $\delta>\beta$.

(iv) $w \sim w_{-}+\mu^{-\delta}$ where $\delta>\beta$.

The type (ii) limit yields an infinite curve $C_{R}$ given by $y_{i}^{N_{f}-N_{c}-i} x_{i}^{N_{f}-N_{c}-i+1}=1$. The type (i) limit yields components $C_{i=1, \ldots, N_{c}-1}$ with the multiplicity of $C_{i}$ being $\left(N_{c}-i\right)$. The type (iii) limit yields components $C_{i=r+1, \ldots, r_{*}-1}$ with multiplicity $(i-r)$ if $r<r_{*}$, while it yields components $C_{i=r_{*}+1, \ldots, r-1}$ with multiplicity $(r-i)$ if $r>r_{*}$. The type (iv) limit yields components $C_{i=r_{*}+1, \ldots, N_{f}-r-1}$ with multiplicity $\left(N_{f}-r-i\right)$. These components are all at $w=0$. In the first patch, there is another component $C_{L}$ given by $y_{1}=w^{N_{c}}, x_{1}=0$.

Together with the components which already exist before the rotation, the $\mu \rightarrow \infty$ limit of the curve for the $r$-th non-baryonic branch root is the same as the limit of the curve for the baryonic branch root which is depicted in figure 11 .

\section{More on the $N=1$ Moduli Space of Vacua}

In this section we will discuss the moduli space of vacua of the $N=1$ theories for finite as well as infinite adjoint mass $\mu$ including the baryonic degrees of freedom. 


\subsection{M-theory Proof of the $s$-Rule}

We have seen in the previous sections that for finite adjoint mass the moduli space of vacua consists of non-baryonic branches parametrized by an integer $r$ and having complex dimension $2 r\left(N_{f}-r\right)$ and a baryonic branch of complex dimension $2 N_{c} N_{f}-2\left(N_{c}^{2}-1\right)$ which exists when $N_{f} \geq N_{c}$. In the limit $\mu \rightarrow \infty$ these different branches become submanifolds of the Higgs branch of $N=1 \mathrm{SQCD}$. The complex dimension of the Higgs branch of $N=1 \mathrm{SQCD}$ is $2 N_{c} N_{f}-\left(N_{c}^{2}-1\right)$ which means that in the limit $\mu \rightarrow \infty$ an extra $N_{c}^{2}-1$ degrees of freedom become massless. We will start by showing how this can be seen in the M-theory fivebrane picture.

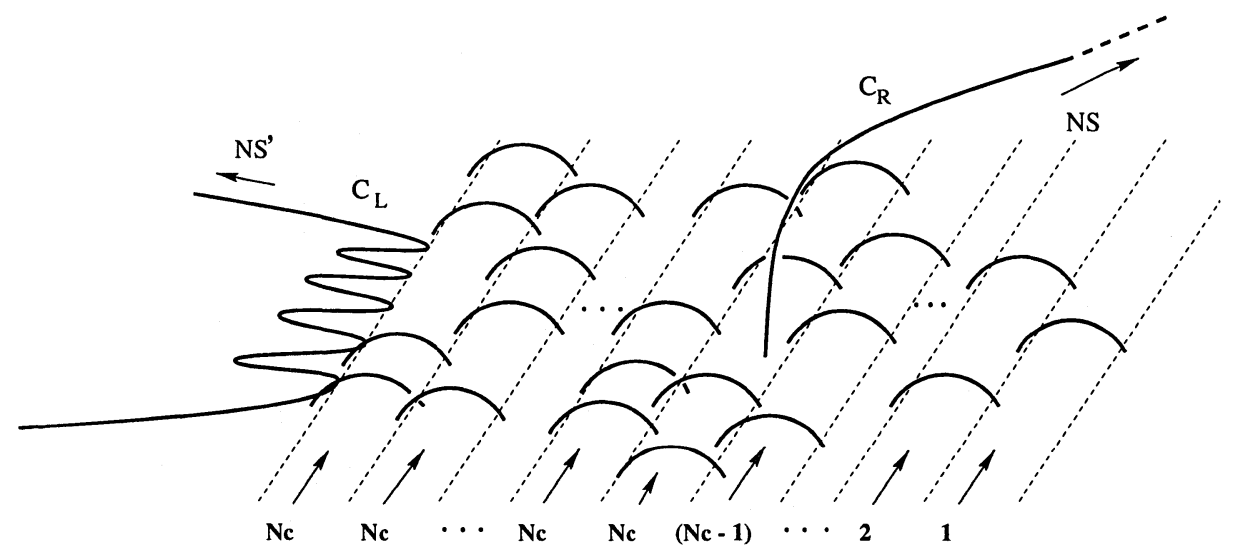

Figure 14: M-Theory description of the Higgs branch.

As in previous cases we have to describe the curve in the resolved $A_{N_{f}-1}$ surface. This is depicted in figure 14. The left component $C_{L}$ of the curve describing the NS' 5 -brane is given by

$$
\tilde{t}=w^{N_{c}}, \quad v=0,
$$

while the right component $C_{R}$ of the curve describing the NS 5-brane is given by

$$
\widetilde{t}=\Lambda_{N=1}^{3 N_{c}-N_{f}} v^{N_{f}-N_{c}}, \quad w=0 .
$$

Possible deformations of the left component (6.1) are

$$
\tilde{t}=w^{N_{c}}+s_{1} w^{N_{c}-1}+\ldots+s_{N_{c}}, \quad v=0 .
$$

It is not possible to deform the right component (6.2). This follows from the invariance under the action of $U(1)_{4,5}$ and $U(1)_{8,9}$, where the charges are given in (5.5). Since there is no order parameter carrying only $U(1)_{4,5}$ charges this symmetry cannot be broken and the equation (6.2) is fixed, while 
the vev of the meson field can break the $U(1)_{8,9}$ symmetry and corresponds to the deformation (6.3). The fact that there are no allowed deformations of (6.2) can also be deduced in the case $N_{f} \geq 1$ by the following argument. Suppose that we try to deform it by adding other monomials in $v$. This implies that there will be non-zero values of $v$ for which $\widetilde{t}=0$. Recall, however, that the $(\tilde{t}, v)$ coordinates are of the Taub-NUT space

$$
\tilde{t} x=\Lambda_{N=1}^{3 N_{c}-N_{f}} v^{N_{f}},
$$

which excludes $\widetilde{t}=0$ for non-zero values of $v$. Therefore deformations of (6.2) are not allowed. This in fact provides a proof of the $s$-rule.

To see that this indeed corresponds to the $s$-rule, let us compare IIA and M-theory brane configurations. The Higgs branch of $N=1 \mathrm{SQCD}$ is depicted in figure 15. For comparison with the M-theory description of the

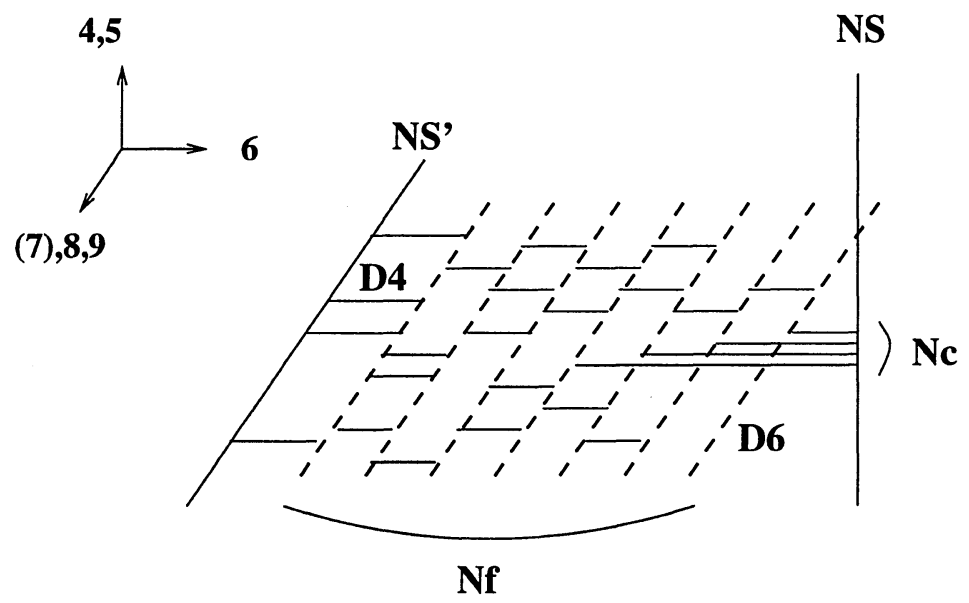

Figure 15: Type IIA description of the Higgs branch.

Higgs branch it is useful to move the right NS 5-brane in figure 15 and pass $N_{c}$ D6-branes. The configuration that we end with is plotted in figure 16 .

The $\mathbb{C P}^{1}$ components in figure 14 correspond to the $\mathrm{D} 4$-branes suspended between the D6-branes in the IIA picture of figure 16. The $N_{c}$ complex moduli associated with the $N_{c}$ D4-branes suspended between the NS' 5 -brane and the D6-brane to its left are seen in the M-theory fivebrane framework as deformations of the left part of the curve in figure 14 which is the part of the curve describing the NS' 5 -brane. The existence of allowed deformations of the left part of the curve in figure 14 that describes the NS' 5-brane and the non-existence of allowed deformations of the right part of the curve in figure 14 that describes the NS 5-brane is from the IIA viewpoint the $s$-rule which has been suggested empirically in [5]. 


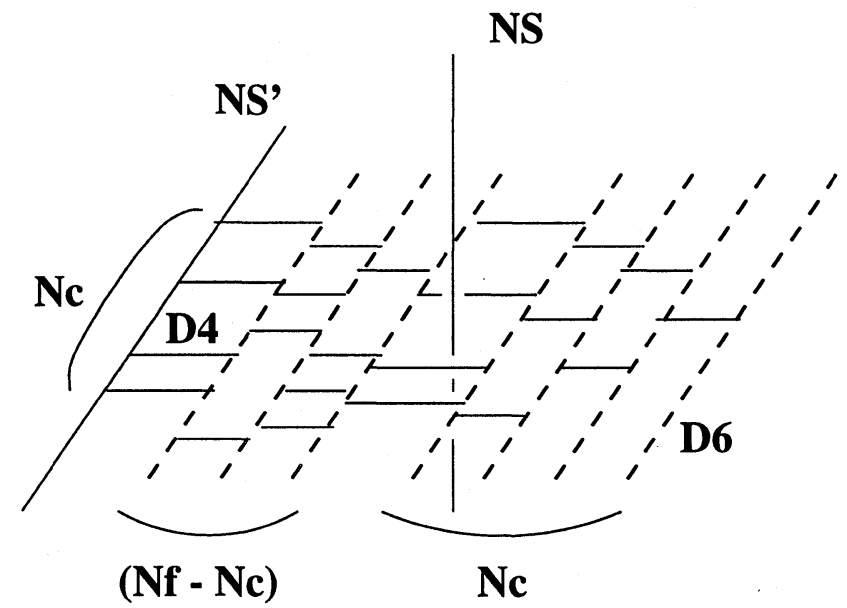

Figure 16: Alternative type IIA description of the Higgs branch.

Here we have shown that (6.3) are the only permissible deformations of the left and the right curves, consistent with the asymptotic conditions and the $U(1)$ R-symmetry. To show that all these deformations actually correspond to vevs of fields in the $N=1$ theory, we need to compute the Kähler metric for these deformations and show that it is regular and nondegenerate. This issue is currently under investigation [42].

A simple counting of the number of D4-branes suspended between the D6-branes and between the D6-branes and the NS' 5 -brane gives $\left(2 N_{c} N_{f}-\right.$ $N_{c}^{2}$ ) as the complex dimension of the $N=1$ theory. As in the $N=2$ case the type IIA brane counting results in the dimension of a $U\left(N_{c}\right)$ gauge group instead of an $S U\left(N_{c}\right)$ gauge group, missing one complex dimensions in the moduli space. It is possible that this missing dimension corresponds to the relative locations of the NS and NS' 5 -branes in the $x^{7}$-direction and its superpartner. In order to fully establish this, we need to compute the Kähler metric for this direction.

\subsection{The Baryonic Degrees of Freedom}

In section 4 we showed how the meson matrix is realized in the M-theory fivebrane. For $N_{f} \geq N_{c}$ there are also baryonic degrees of freedom. In the following we will show how the latter are realized in the M-theory fivebrane. We will derive the equations (2.29), (2.38) and (2.41) of section 2 from the fivebrane.

We start with the case $N_{f}=N_{c}$. The vev for the baryon operators is zero on the non-baryonic branches and is non zero on the baryonic branch. In order to illustrate the difference between the non-baryonic and baryonic 
branches let us consider the $r$-th non-baryonic branch with $r=\frac{N_{f}}{2}$. In the limit $\mu \rightarrow \infty$ it is described by (5.23)

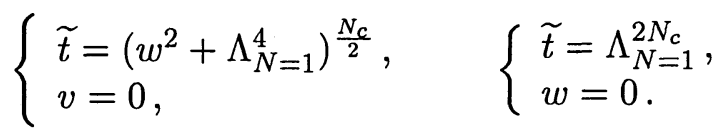

In figure 17 we plot for simplicity the two components of the curve (6.5) in the case $N_{c}=N_{f}=2$. The above non-baryonic branch is part of the $N=1$ SQCD Higgs branch where $\tilde{B} B=0$.

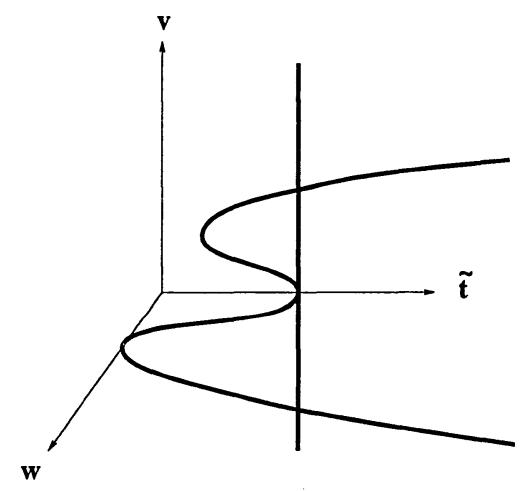

Figure 17: The two components of (6.5) for $N_{c}=N_{f}=2$.

For comparison consider now the baryonic branch in the limit $\mu \rightarrow \infty$. It is given by $(5.25)$

$$
\left\{\begin{array}{l}
\tilde{t}=w^{N_{c}}, \quad\left\{\begin{array}{l}
\tilde{t}=\Lambda_{N=1}^{2 N_{c}}, \\
v=0,
\end{array}, 0 .\right.
\end{array}\right.
$$

In figure 18 we plot the two components of (6.6). The baryonic branch is part of the $N=1$ SQCD Higgs branch where $\tilde{B} B \neq 0$.

Comparing figures 17 and 18 we see that while in figure 17 the two branches intersect, in figure 18 they do not. The distance between the two branches at $w=0$ is a candidate for $\tilde{B} B$, when the vev of the meson matrix vanishes. As a first check we have to see that it carries the $U(1)_{R}$ and $U(1)_{A}$ charges of $\tilde{B} B$. Using (5.5) and the relation between the $U(1)_{R}$ and $U(1)_{A}$ charges and the $U(1)_{45}$ and $U(1)_{89}$ charges:

$$
\begin{aligned}
U(1)_{R} & =\frac{N_{c}}{N_{f}} U(1)_{45}+\frac{N_{f}-N_{c}}{N_{f}} U(1)_{89} \\
U(1)_{A} & =-U(1)_{45}+U(1)_{89},
\end{aligned}
$$

we see that $\tilde{t}$ carries the charges $\left(U(1)_{R}, U(1)_{A}\right)=\left(2 N_{c} \frac{N_{f}-N_{c}}{N_{f}}, 2 N_{c}\right)$ which are the correct charges that $\tilde{B} B$ carries. 


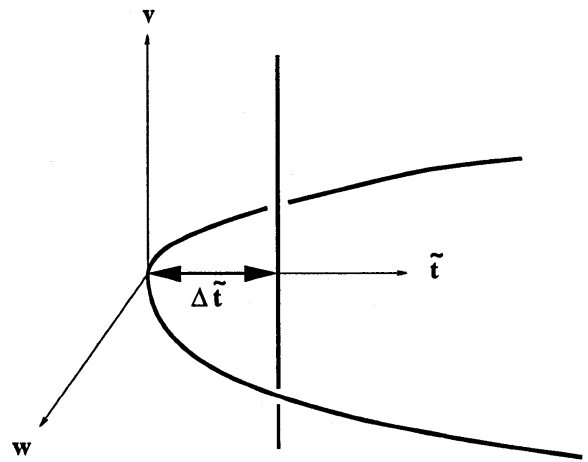

Figure 18: The two components of (6.6).

A non-trivial check is to verify that when we shift the second branch of (6.6) by shifting $w$ as in figure 19 which means giving a vev for the meson, the values of the distance and the shift have to satisfy the relation (2.29).

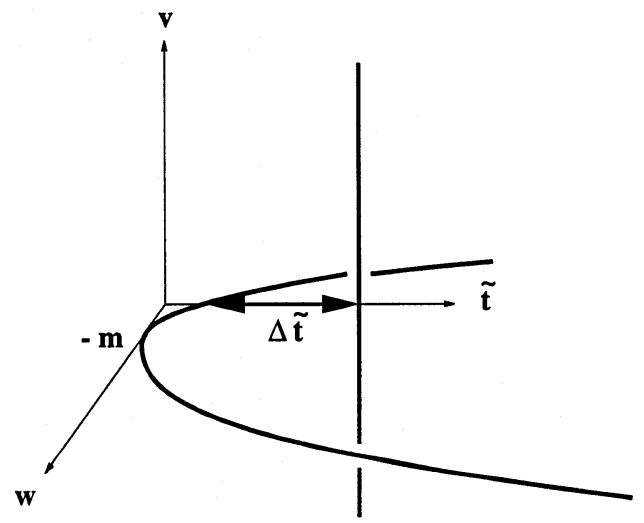

Figure 19: Shifting $w$ in (6.6).

Indeed we see that the distance $\Delta \widetilde{t}$ between the two branches at $w=0$ is

$$
m^{N_{c}}-\Delta \widetilde{t}=\Lambda_{N=1}^{2 N_{c}},
$$

which is identical to $(2.29)$ if we identify the distance $\Delta \tilde{t}$ as $\tilde{B} B$. Note also, that the brane equation (6.8) captures the fact that in $N=1$ SQCD with $N_{f}=N_{c}$ the classical moduli space of vacua is modified quantum mechanically.

Consider next the case $N_{f}=N_{c}+1$, and let $\mu$ be finite. The two components of the curve corresponding to the baryonic branch are given by 
(5.24). As in the case $N_{f}=N_{c}$ we shift $w \rightarrow w+m$ and get

$$
\left\{\begin{array} { l } 
{ \tilde { t } = ( w + m ) ^ { N _ { c } } , } \\
{ v = \mu ^ { - 1 } ( w + m ) , }
\end{array} \quad \left\{\begin{array}{l}
\tilde{t}=\Lambda_{N=1}^{3 N_{c}-N_{f}} v, \\
w=0
\end{array}\right.\right.
$$

It is easy to compute the distance $\Delta \widetilde{t}$ between the two components of the curve (6.9) at $w=0$ and we get

$$
m^{N_{c}}-\Delta \widetilde{t}=\Lambda_{N=1}^{3 N_{c}-N_{f}} \frac{m}{\mu}
$$

which is identical to (2.38) if we identify the distance $\Delta \tilde{t}$ as $\tilde{B} B$. Note however that there is a difference between the $N_{f}=N_{c}+1$ and $N_{f}=N_{c}$ cases. In the former $\tilde{B} B$ is a matrix while in the latter it has a single component. Clearly the distance between the two parts of the baryonic branch curve cannot provide us with the information on the full $\tilde{B} B$ matrix. Equations (6.10) and (2.38) describe only part of the possible vev's for the baryons. In the limit $\mu \rightarrow \infty$ the RHS of (6.10) vanishes and we get the description of the moduli space of vacua of $N=1 \mathrm{SQCD}$ with $N_{f}=N_{c}+1$. In this case, the classical moduli space of vacua is not modified quantum mechanically.

Finally, let $N_{f}>N_{c}+1$, and keep $\mu$ finite. In this case there was an ambiguity in the field theory analysis in finding the extrema of the superpotential in section 2. The two components of the curve corresponding to the baryonic branch read now

$$
\left\{\begin{array} { l } 
{ \tilde { t } = ( w + m ) ^ { N _ { c } } , } \\
{ v = \mu ^ { - 1 } ( w + m ) , }
\end{array} \quad \left\{\begin{array}{l}
\tilde{t}=\Lambda_{N=1}^{3 N_{c}-N_{f}} v^{N_{f}-N_{c}}, \\
w=0,
\end{array}\right.\right.
$$

where we shifted $w \rightarrow w+m$. The distance $\Delta \widetilde{t}$ between the two components of the curve (6.11) at $w=0$ satisfies

$$
m^{N_{c}}-\Delta \tilde{t}=\mu^{N_{c}-N_{f}} m^{N_{f}-N_{c}} \Lambda_{N=1}^{3 N_{c}-N_{f}} .
$$

This relation is identical to (2.41) which we derived by field theory means with one choice of approaching the region in moduli space where the determinant of the meson matrix vanishes. Unlike the field theory analysis, there is no ambiguity in the brane framework in deriving (6.11). This is not surprising since we expect the brane to provide us with the good coordinates on the moduli space of vacua. The ambiguity that we encountered in section 2 can be traced to the fact that $\operatorname{det} M=0$ defines a moduli space and we need information about the good coordinates near that region in order to approach it. As in the previous case, in the limit $\mu \rightarrow \infty$ the RHS of (6.12) vanishes and we get the correct description of the moduli space of vacua of 
$N=1$ SQCD with $N_{f}>N_{c}+1$, where the classical moduli space is not modified quantum mechanically.

To summarize: We showed in this section how the baryonic degrees of freedom (up to the chiral rotation) are realized in M-theory fivebrane. In particular we found a complete agreement for both finite and infinite values of the adjoint mass between the field theory results of section 2 and the fivebrane description of the moduli space of vacua.

\section{Beyond Holomorphy - Kähler Potential}

We have shown that the configuration of the fivebrane encodes strong coupling physics of the $N=1$ theory, in particular the Affleck-Dine-Seiberg superpotential for $N_{c}>N_{f}$ and the holomorphic structure of the moduli space for $N_{c} \leq N_{f}$. In the field theory approach, these non-perturbative results were obtained using the holomorphy argument. In order to fully understand the low energy dynamics, however, we also need to determine the Kähler potential. In the $N=1$ theory, the Kähler potential is independent of the superpotential and the holomorphy argument is not sufficient to specify it.

We expect, in this regard, the fivebrane approach to be more powerful than the standard field theory method. The eleven-dimensional Planck scale $l_{11}$, the radius $R$ of the eleventh dimensional circle, the type IIA string coupling constant $g_{s}$ and the string scale $l_{s}$ are related as

$$
l_{11} \sim g_{s}^{1 / 3} l_{s}, \quad R \sim g_{s} l_{s} .
$$

On the other hand, the gauge coupling constant $g_{\text {gauge }}$ of $N=1$ theory that arises from the web of branes scales [5] as

$$
g_{\text {gauge }}^{2} \sim \frac{g_{s} l_{s}}{L_{b r a n e}},
$$

where $L_{\text {brane }}$ is the distance between the NS and NS' 5 -branes. Therefore

$$
g_{\text {gauge }} \sim\left(\frac{R}{L_{\text {brane }}}\right)^{1 / 2} .
$$

This means that we can take the limit $R / l_{11}, L_{\text {brane }} / l_{11} \rightarrow \infty$ while keeping $g_{\text {gauge }}$ finite, and the low energy effective theory of M-theory, namely the eleven-dimensional supergravity, should give an exact description of $N=1$ theory. Low energy degrees of freedom of the fivebrane are its deformation in the spacetime and the chiral anti-symmetric tensor field on the brane. Thus, we can directly read off their kinetic terms from the fivebrane action [43] and find the Kähler metric. 
One of the interesting questions concerning the Kähler potential is its behavior at the origin of the moduli space for $N_{c}<N_{f}$. If one can show, for example that the Kähler metric is regular at the origin for $N_{f}=N_{c}+1$, it gives a direct confirmation of the claim (based on the 't Hooft anomaly matching condition) that both the mesons and the baryons are massless degrees of freedom there. Since the superpotential for $N_{f}=N_{c}+1$ is given by

$$
W=\Lambda^{1-2 N_{c}}(\tilde{B} M B-\operatorname{det} M),
$$

if the metric $g_{i \bar{j}}$ is regular, the potential for the scalar fields

$$
V=g^{i \bar{j}} \partial_{i} W \bar{\partial}_{\bar{j}} \bar{W}
$$

vanishes quartically and is flat at the origin. We hope to discuss more on this subject in our future publication [42].

\section{Note added}

After completing this work we were informed of [44] where related issues are studied.

\section{Acknowledgements}

We would like to thank D. Kutasov, H. Murayama, R. Plesser, C. Vafa and E. Witten for valuable discussions. K.H. would like to thank Institute for Advanced Study and Rutgers Physics Department, H.O. would like to thank Harvard and Rutgers Physics Departments and Y.O. would like to thank the Weizmann Institute Physics Department, for hospitality.

This research is supported in part by NSF grant PHY-95-14797 and DOE grant DE-AC03-76SF00098.

\section{References}

[1] J. Polchinski, Phys. Rev. Lett. 75 (1995) 4724.

[2] S. Kachru and C. Vafa, Nucl. Phys. B450 (1995) 69.

[3] S. Kachru, A. Klemm, W. Lerche, P. Mayr and C. Vafa, Nucl. Phys. B459 (1996) 537.

[4] A. Klemm, W. Lerche, P. Mayr, C. Vafa and N. Warner, Nucl. Phys. B477 (1996) 746.

[5] A. Hanany and E. Witten, Nucl. Phys. B492 (1997) 152.

[6] J. M. Maldacena, "Black Holes in String Theory", Princeton University thesis, hep-th/9607235. 
[7] J. L. F. Barbon, Phys. Lett. B402 (1997) 59.

[8] S. Elitzur, A. Giveon and D. Kutasov, Phys. Lett. B400 (1997) 269.

[9] S. Elitzur, A. Giveon, E. Rabinovici, A. Schwimmer and D. Kutasov, "Brane Dynamics and N=1 Supersymmetric Gauge Theory", hep-th/9704104.

[10] E. Witten, "Solutions of Four-Dimensional Field Theories Via M-Theory", hep-th/9703166.

[11] K. Becker, M. Becker and A. Strominger, Nucl. Phys. B456 (1995) 130.

[12] E. Verlinde, Nucl. Phys. B455 (1995) 211.

[13] N. Evans, C. V. Johnson and A. D. Shapere, "Orientifolds, Branes, and Duality of 4D Gauge Theories", hep-th/9703210.

[14] N. Seiberg and E. Witten, Nucl. Phys. B426 (1994) 19; Nucl. Phys. B431 (1994) 484.

[15] A. Klemm, W. Lerche, S. Theisen and S. Yankielowicz, Phys. Lett. B344 (1995) 169.

[16] P. Argyres and A. Faraggi, Phys. Rev. Lett. 74 (1995) 3931.

[17] A. Hanany and Y. Oz, Nucl. Phys. B452 (1995) 283.

[18] P. C. Argyres, M. R. Plesser and A. D. Shapere, Phys. Rev. Lett. 75 (1995) 1699.

[19] I. Affleck, M. Dine and N. Seiberg, Nucl. Phys. B241 (1984) 493.

[20] N. Seiberg, Phys. Rev. D49 (1994) 6857.

[21] N. Seiberg, Nucl. Phys. B435 (1995) 152.

[22] J. de Boer, K. Hori, H. Ooguri, Y. Oz and Z. Yin, Nucl. Phys. B493 (1997) 148.

[23] J. de Boer, K. Hori, Y. Oz and Z. Yin, "Branes and Mirror Symmetry in $N=2$ Supersymmetric Gauge Theories in Three Dimensions", hep-th 9702154.

[24] H. Ooguri and C. Vafa, "Geometry of $N=1$ Dualities in Four Dimensions", hep-th/9702180.

[25] C. Ahn and K. Oh, "Geometry, D-Branes and N=1 Duality in Four Dimensions I", hep-th/9704061.

[26] J. H. Brodie and A. Hanany, "Type IIA Superstrings, Chiral Symmetry, and N=1 4D Gauge Theory Dualities", hep-th/9704043.

[27] A. Brandhuber, J. Sonnenschein, S. Theisen and S. Yankielowicz, "Brane Configurations and 4D Field Theory Dualities", hep-th/9704044.

[28] O. Aharony and A. Hanany, "Branes, Superpotentials and Superconformal Fixed Points", hep-th/9704170.

[29] C. Ahn, "Geometry, D-Branes and N=1 Duality in Four Dimensions II", hepth/9705004.

[30] R. Tatar, "Dualities in 4D Theories with Product Gauge Groups from Brane Configurations", hep-th/9704198. 
[31] I. Brunner and A. Karch, "Branes and Six Dimensional Fixed Points", hepth/9705022.

[32] B. Kol, "5d Field Theories and M-Theory", hep-th/9705031.

[33] C. Ahn and R. Tatar, "Geometry, D-branes and N=1 Duality in Four Dimensions with Product Gauge Group", hep-th/9705106.

[34] K. Landsteiner, E. Lopez and D. A. Lowe, " $N=2$ Supersymmetric Gauge Theories, Branes and Orientifolds", hep-th/9705199.

[35] A. Brandhuber, J. Sonnenschein, S. Theisen and S. Yankielowicz, " $M-T h e o r y$ And Seiberg-Witten Curves: Orthogonal and Symplectic Groups", hepth/9705232.

[36] A. Hanany and A. Zaffaroni, "Chiral Symmetry from Type IIA Branes", hepth/9706047.

[37] E. Witten, Nucl. Phys. B202 (1982) 253.

[38] P. C. Argyres, M. R. Plesser and N. Seiberg, Nucl. Phys. B471 (1996) 15.

[39] K. Intriligator and N. Seiberg, Nucl. Phys. Proc. Suppl. 45BC (1996) 1.

[40] P. K. Townsend, Phys. Lett. B350 (1995) 184.

[41] K. Hori, H. Ooguri and C. Vafa, "Non-Abelian Conifold Transitions and $N=4$ Dualities in Three Dimensions", hep-th/9705220.

[42] K. Hori, H. Ooguri and Y. Oz, work in progress.

[43] For the current status of the subject, see for example, J. H. Schwarz, "The M-Theory Five-Brane and the Heterotic String", hep-th/9705092.

[44] E. Witten, "Branes And The Dynamics Of QCD", hep-th/9706109. 